\title{
Uniqueness of the Optimal Nodes of Quadrature Formulae
}

\author{
By Borislav D. Bojanov
}

\begin{abstract}
We prove the uniqueness of the quadrature formula with minimal error in the space $\tilde{W}_{q}^{r}[a, b], 1<q<\infty$, of $(b-a)$-periodic differentiable functions among all quadratures with $n$ free nodes $\left\{x_{k}\right\}_{1}^{n}, a=x_{1}<\cdots<x_{n}<b$, of fixed multiplicities $\left\{v_{k}\right\}_{1}^{n}$, respectively. As a corollary, we get that the equidistant nodes are optimal in $\tilde{W}_{q}^{\prime}[a, b]$ for $1<q<\infty$ if $\nu_{1}=\cdots=\nu_{n}$.
\end{abstract}

1. Introduction. Let $\mathscr{F}$ be a given class of sufficiently smooth functions defined on the interval $[a, b]$. Suppose that the linear functional $L(f)$ is defined on $\mathscr{F}$. We shall consider in this paper rules of the type

$$
L(f) \approx \sum_{k=1}^{n} \sum_{\lambda=0}^{\nu_{k}-1} a_{k \lambda} f^{(\lambda)}\left(x_{k}\right)=: S(\mathbf{a}, \mathbf{x} ; f)
$$

with nodes

$$
\mathbf{x}=\left(\begin{array}{c}
x_{1} \ldots x_{n} \\
\nu_{1} \ldots \nu_{n}
\end{array}\right), \quad a \leqslant x_{1}<\cdots<x_{n}<b,
$$

and coefficients $\mathbf{a}=\left\{a_{k \lambda}\right\}$. Let $R(\mathbf{a}, \mathbf{x}):=\sup \{|L(f)-S(\mathbf{a}, \mathbf{x} ; f)|: f \in \mathscr{F}\}$ denote the error of the rule (1.1) in the class $\mathscr{F}$.

Definition 1. The coefficients $\mathbf{a}(\mathbf{x})$ are said to be best for the nodes $\mathbf{x}$ if

$$
\begin{aligned}
R(\mathbf{a}(\mathbf{x}), \mathbf{x}) & =\inf \left\{R(\mathbf{b}, \mathbf{x}): \text { over all real } \mathbf{b}=\left\{b_{k \lambda}\right\}\right\} \\
& =: R\left(x_{1}, \ldots, x_{n}\right) .
\end{aligned}
$$

We shall refer to (1.1) as being a best rule if its coefficients $\left\{a_{k \lambda}\right\}$ are best for the nodes $\mathbf{x}$.

Definition 2. We call the nodes $\left\{x_{k}\right\}_{1}^{n}$ optimal of type $\left(\nu_{1}, \ldots, \nu_{n}\right)$ in the class $\mathscr{F}$ if $x_{1}<\cdots<x_{n}$ and

$$
R\left(x_{1}, \ldots, x_{n}\right)=\inf \left\{R\left(y_{1}, \ldots, y_{n}\right): a<y_{1}<\cdots<y_{n}<b\right\} .
$$

The rule (1.1) with nodes $\mathbf{x}$ defined by (1.3) and coefficients $\mathbf{a}(\mathbf{x})$ is optimal of type $\left(\nu_{1}, \ldots, \nu_{n}\right)$.

The main purpose of this paper is to prove the uniqueness of the optimal nodes of arbitrary fixed type $\left(\nu_{1}, \ldots, \nu_{n}\right)$ for $L(f)=\int_{a}^{b} f(t) d t$ in the Sobolev spaces $\tilde{W}_{q}^{r}[a, b], 1<q<\infty$,

$$
\begin{aligned}
& \tilde{W}_{q}^{r}[a, b]:=\left\{(b-a) \text {-periodic } f: f \in C^{r-1}(-\infty, \infty),\right. \\
& \left.\qquad f^{(r-1)} \text { locally abs. cont., }\left\|f^{(r)}\right\|_{q}<\infty\right\},
\end{aligned}
$$

Received December 21, 1979; revised July 10, 1980.

1980 Mathematics Subject Classification. Primary 65D30; Secondary 41 A55. 
where

$$
\begin{aligned}
& \|f\|_{q}:=\left\{\int_{a}^{b}|f(t)|^{a} d t\right\}^{1 / q} \text { for } 1<q<\infty, \\
& \|f\|_{\infty}:=\operatorname{supvrai}\{|f(t)|: t \in[a, b]\} .
\end{aligned}
$$

The error $R(\mathbf{a}, \mathbf{x})$ of the rule (1.1) is defined in this case by

$$
R(\mathbf{a}, \mathbf{x})=\sup \left\{|L(f)-S(\mathbf{a}, \mathbf{x} ; f)|: f \in \tilde{W}_{q}^{r}[a, b],\left\|f^{(r)}\right\|_{q}<1\right\} .
$$

As an immediate consequence of our uniqueness theorem we get that the equidistant nodes are optimal of type $\left(\nu_{1}, \ldots, \nu_{n}\right)$ in $\tilde{W}_{q}^{r}[a, b]$ for $1<q<\infty$ if the multiplicities $\left\{\nu_{k}\right\}_{1}^{n}$ are equal. Considerable effort was expended, in the last years, on the proof of this intuitively obvious fact. It should be pointed out that particular cases of the consequence mentioned have been given by many authors, see [14], [18], [19], [10], [12]. Recently, Žensykbaev [20] showed the optimality of the equidistant nodes in the simple node case, i.e., when $\nu_{1}=\cdots=\nu_{n}=1$ for $1 \leqslant q \leqslant \infty$.

Using the well-known one-to-one correspondence between quadrature formulae and monosplines, one can restate our main result as a uniqueness theorem for the extremal problem $\|M\| \rightarrow$ inf, $1<p<\infty$, over all $(b-a)$-periodic monosplines of degree $r$ with free knots $\left\{x_{k}\right\}_{1}^{n}$ of fixed multiplicities $\left\{\nu_{k}\right\}_{1}^{n}$, respectively.

In Section 2 we present some results which characterize the best rule and may be used to calculate the best coefficients $\mathbf{a}(\mathbf{x})$. Section 3 contains our main uniqueness theorem. In order to facilitate the presentation of the results, we have deferred the proofs of all auxiliary lemmas to an appendix.

2. Preliminaries. Throughout we will assume that the multiplicities $\left\{\nu_{k}\right\}_{1}^{n}$ are natural numbers satisfying the inequalities $1<\nu_{k} \leqslant r, k=1, \ldots, n, n>1$. We shall write

$$
\mathbf{x}=\left(\begin{array}{c}
x_{1} \ldots x_{n} \\
\nu_{1} \ldots \nu_{n}
\end{array}\right)
$$

to indicate that $\mathbf{x}$ is a system of points $\left\{x_{k}\right\}_{1}^{n}$ of multiplicities $\left\{\nu_{k}\right\}_{1}^{n}$, respectively, and such that $a=x_{1}<\cdots<x_{n}<b$.

Given the points $\left\{y_{k}\right\}_{1}^{n}, a=y_{1}<y_{2}<\cdots<y_{n}<b$, and (2.1) we define the function $M_{\alpha}(t)$ on $[a, b]$ by

$$
\begin{aligned}
M_{\alpha}(t)= & \alpha \frac{(b-t)^{r}}{r !}+(1-\alpha) \sum_{k=2}^{n} \frac{\left(y_{k}-t\right)_{+}^{r-1}}{(r-1) !}+\sum_{i=0}^{r-1} \beta_{i} \frac{(b-t)^{r-i-1}}{(r-i-1) !} \\
& -\sum_{k=2}^{n} \sum_{\lambda=0}^{\nu_{k}-1} a_{k \lambda} \frac{\left(x_{k}-t\right)_{+}^{r-\lambda-1}}{(r-\lambda-1) !}
\end{aligned}
$$

where $\alpha$ is a parameter from $[0,1]$ and $\left\{\beta_{i}\right\},\left\{a_{k \lambda}\right\}$, are real numbers. In the following discussion we shall assume that $M_{\alpha}$ satisfies the boundary conditions

$$
M_{\alpha}^{(j)}(a+)=M_{\alpha}^{(j)}(b-), \quad j=0, \ldots, r-\nu_{1}-1
$$

Remark 1. It is best to think of $M_{\alpha}$ as being defined on a circle obtained by joining the endpoints $a$ and $b$ of the interval $[a, b]$. Then $M_{\alpha}$ may be considered to be a periodic monospline of degree $r$ with simple knots $y_{2}, \ldots, y_{n}$ and multiple knots $\left\{x_{k}\right\}_{1}^{n}$ with corresponding multiplicities $\left\{\nu_{k}\right\}_{1}^{n}$. 
If $f \in \tilde{W}_{\mathrm{I}}^{r}[a, b]$, then integration by parts produces the identity

$$
\begin{aligned}
\int_{a}^{b} M_{\alpha}(t) f^{(r)}(t) d t= & \alpha \int_{a}^{b} f(t) d t+(1-\alpha) \sum_{k=2}^{n} f\left(y_{k}\right) \\
& -\sum_{k=1}^{n} \sum_{\lambda=0}^{\nu_{k}-1} a_{k \lambda} f^{(\lambda)}\left(x_{k}\right),
\end{aligned}
$$

where $\left\{a_{k \lambda}, k=2, \ldots, n, \lambda=0, \ldots, \nu_{k}-1\right\}$ are the same as in (2.2) and

$$
\begin{aligned}
& a_{1 \lambda}=(-1)^{r-1-\lambda}\left\{M_{\alpha}^{(r-1-\lambda)}(b-)-M_{\alpha}^{(r-1-\lambda)}(a+)\right\} \\
& \lambda=0, \ldots, \nu_{1}-1 .
\end{aligned}
$$

The relation (2.4) suggests a method of approximation of the functional

$$
L_{\alpha}(f):=\alpha \int_{a}^{b} f(t) d t+(1-\alpha) \sum_{k=2}^{n} f\left(y_{k}\right)
$$

by a linear expression using the values $\left\{f^{(\lambda)}\left(x_{k}\right)\right\}$. Moreover, the error of this method at every $f$ is given by

$$
R_{\alpha}(f)=\int_{\alpha}^{b} M_{\alpha}(t) f^{(r)}(t) d t .
$$

It is clear that $R_{\alpha}(f)=0$ for every $f \in \tilde{W}_{1}^{r}[a, b]$ with $f^{(r)}(t) \equiv 0$, i.e., for $f=$ const. Conversely, every linear method of the form $L_{\alpha}(f) \approx S(\mathbf{a}, \mathbf{x} ; f)$ which is exact for each $f=$ const has an error expression (2.6) with $M_{\alpha}(t)$ satisfying (2.3). This is a one-to-one correspondence between monosplines (2.2) and rules (1.1) which we shall use in the sequel.

In the following we discuss the construction of the best rule for fixed $\mathbf{x}$ in the case $\mathscr{F}=\tilde{W}_{q}^{r}[a, b], L=L_{\alpha}$. Since $L_{\alpha}(f)-S(\mathbf{a}, \mathbf{x} ; f)=\int_{a}^{b} M_{\alpha}(t) f^{(r)}(t) d t$, application of Hölder's inequality shows that the extremal problem (1.2) is equivalent to the following one

$$
\left\|M_{\alpha}\right\|_{p} \rightarrow \text { minimum, } \quad 1 / p+1 / q=1,
$$

over all $M_{\alpha}$ satisfying (2.3). But (2.7) is a classical approximation problem in normed linear space (see [1, p. 17]). It has a unique solution for $1<p<\infty$. Let us denote it by $M_{\alpha}(\mathbf{x} ; t)$. The parameter $p$ is fixed and so is not mentioned explicitly in the notation.

The next theorem presents a characterization of the extremal element $M_{\alpha}(\mathbf{x} ; t)$.

Theorem 1. Let $1<p<\infty$ and $\alpha \in[0,1]$. Suppose that $r$ and $\mathbf{x}$ are given. The function $M_{\alpha}(t)$ is a solution of the extremal problem (2.7) if and only if there exists a system of numbers $\left\{a_{i}\right\}_{0}^{r-1}$ such that

$$
\begin{cases}F_{\alpha}^{(j)}(a)=F_{\alpha}^{(j)}(b), & j=0, \ldots, r-1, \\ F_{\alpha}^{(\lambda)}\left(x_{k}\right)=0, & k=1, \ldots, n, \lambda=0, \ldots, \nu_{k}-1, \\ M_{\alpha}^{(j)}(a+)=M_{\alpha}^{(j)}(b-), & j=0, \ldots, r-\nu_{1}-1,\end{cases}
$$

where

$$
F_{\alpha}(x)=(p-1) \sum_{i=0}^{r-1}\left(a_{i} / i !\right) x^{i}+\int_{a}^{b} \frac{(x-t)_{+}^{r-1}}{(r-1) !}\left|M_{\alpha}(t)\right|^{p-2} M_{\alpha}(t) d t
$$


Proof. Suppose that $\left\|M_{\alpha}\right\|_{p}$ is minimal. The first necessary conditions for minimum of $\left\|M_{\alpha}\right\|_{p}$ give

$$
\begin{aligned}
\frac{\partial \Psi}{\partial a_{k \lambda}} & =0, \quad k=2, \ldots, n, \lambda=0, \ldots, \nu_{k}-1, \\
\frac{\partial \Psi}{\partial \beta_{i}} & =0, \quad i=0, \ldots, r-1,
\end{aligned}
$$

where

$$
\Psi:=\int_{a}^{b}\left|M_{\alpha}(t)\right|^{p} d t+\sum_{j=0}^{r-\nu_{1}-1} \lambda_{j}\left[M_{\alpha}^{(j)}(b-)-M_{\alpha}^{(j)}(a+)\right]
$$

and $\left\{\lambda_{j}\right\}$ are Lagrange multipliers. Performing the differentiation we get

$$
\begin{aligned}
-p \int_{a}^{b}\left|M_{\alpha}(\mathbf{x} ; t)\right|^{p-2} M_{\alpha}(\mathbf{x} ; t) & \frac{\left(x_{k}-t\right)_{+}^{r-\lambda-1}}{(r-\lambda-1) !} d t \\
& +\sum_{j=0}^{r-\nu_{1}-1}(-1)^{j} \lambda_{j} \frac{\left(x_{k}-a\right)^{r-\lambda-1-j}}{(r-\lambda-1-j) !}=0
\end{aligned}
$$

for $k=2, \ldots, n, \lambda=0, \ldots, \nu_{k}-1$, and

$$
\begin{aligned}
p \int_{a}^{b}\left|M_{\alpha}(\mathbf{x} ; t)\right|^{p-2} M_{\alpha}(\mathbf{x} ; t) & \frac{(b-t)^{r-i-1}}{(r-i-1) !} d t+(-1)^{r-i-1} \lambda_{r-i-1}^{0} \\
& -\sum_{j=0}^{r-\nu_{1}-1}(-1)^{j} \lambda_{j} \frac{(b-a)^{r-i-1-j}}{(r-i-1-j) !}=0
\end{aligned}
$$

for $i=0, \ldots, r-1$, with the stipulation that $t^{\prime} \mid l !=0$ for $l<0$ and

$$
\lambda_{i}^{0}:= \begin{cases}\lambda_{i} & \text { for } i=0, \ldots, r-\nu_{1}-1, \\ 0 & \text { for } i=r-\nu_{1}, \ldots, r-1\end{cases}
$$

Let us set

$$
P_{\alpha}(\mathbf{x} ; t)=\frac{1}{p} \sum_{j=0}^{r-\nu_{1}-1}(-1)^{j+1} \lambda_{j} \frac{(t-a)^{r-1-j}}{(r-1-j) !}
$$

and define the function

$$
F_{\alpha}(\mathbf{x} ; x):=P_{\alpha}(\mathbf{x} ; x)+\int_{a}^{b} \frac{(x-t)_{+}^{r-1}}{(r-1) !}\left|M_{\alpha}(\mathbf{x} ; t)\right|^{p-2} M_{\alpha}(\mathbf{x} ; t) d t
$$

The equations (2.9) and (2.10) show that

$$
\begin{aligned}
F_{\alpha}^{(\lambda)}\left(\mathbf{x} ; x_{k}\right) & =0 \text { for } k=2, \ldots, n, \lambda=0, \ldots, \nu_{k}-1, \\
F_{\alpha}^{(i)}(\mathbf{x} ; b) & =(-1)^{r-i} \lambda_{r-i-1}^{0} / p, \quad i=0, \ldots, r-1 .
\end{aligned}
$$

Further, it is clear from the definition of $F_{\alpha}(\mathbf{x} ; \cdot)$ that

$$
F_{\alpha}^{(i)}(\mathbf{x} ; a)=P_{\alpha}^{(i)}(\mathbf{x} ; a)=(-1)^{r-i} \lambda_{r-i-1}^{0} / p, \quad i=0, \ldots, r-1 .
$$

Therefore

$$
F_{\alpha}^{(i)}(\mathbf{x} ; a)=F_{\alpha}^{(i)}(\mathbf{x} ; b), \quad i=0, \ldots, r-1,
$$


and

$$
F_{\alpha}^{(\lambda)}\left(\mathbf{x} ; x_{1}\right)=0, \quad \lambda=0, \ldots, \nu_{1}-1 .
$$

So, we proved that the function (2.11) satisfies the required conditions.

Now let us prove the sufficiency of the conditions (2.8). Suppose that the numbers $\left\{a_{i}^{*}\right\}_{0}^{r-1}$ and the function $M_{\alpha}^{*}(t)$ with coefficients $\left\{\beta_{i}^{*}\right\},\left\{a_{k \lambda}^{*}\right\}$ satisfy the conditions (2.8). Let $g(t)$ be an arbitrary $(b-a)$-periodic spline function of degree $r-1$ with knots $x=\left(\begin{array}{l}x_{1} \cdots x_{n} \\ v_{1} \cdots v_{n}\end{array}\right)$, i.e., $g(t)$ has a representation of the form

$$
g(t)=\sum_{i=0}^{r-1} \gamma_{i} \frac{(b-t)^{r-i-1}}{(r-i-1) !}-\sum_{k=2}^{n} \sum_{\lambda=0}^{v_{k}-1} c_{k \lambda} \frac{\left(x_{k}-t\right)_{+}^{r-\lambda-1}}{(r-\lambda-1) !}
$$

where $\left\{\gamma_{i}\right\}$ and $\left\{c_{k \lambda}\right\}$ are real coefficients satisfying the conditions

$$
g^{(j)}(a+)=g^{(j)}(b-), \quad j=0, \ldots, r-\nu_{1}-1 .
$$

First we shall show that

$$
\int_{a}^{b}\left|M_{\alpha}^{*}(t)\right|^{p-2} M_{\alpha}^{*}(t) g(t) d t=0 .
$$

Indeed, using (2.9) and (2.10), we get

$$
\begin{aligned}
& \int_{a}^{b}\left|M_{\alpha}^{*}(t)\right|^{p-2} M_{\alpha}^{*}(t) g(t) d t \\
&=\sum_{i=0}^{r-1} \frac{\gamma_{i}}{p}\left[(-1)^{r-i} \lambda_{r-i-1}^{0}+\sum_{j=0}^{r-\nu_{1}-1}(-1)^{j} \lambda_{j} \frac{(b-a)^{r-i-1-j}}{(r-i-1-j) !}\right] \\
&+\sum_{k=2}^{n} \sum_{\lambda=0}^{\nu_{k}-1} \frac{c_{k \lambda}}{p} \sum_{j=0}^{r-\nu_{1}-1}(-1)^{j+1} \lambda_{j} \frac{\left(x_{k}-a\right)^{r-\lambda-1-j}}{(r-\lambda-1-j) !} \\
&= \frac{1}{p}\left\{\sum_{j=0}^{r-\nu_{1}-1} \lambda_{j} g^{(j)}(a+)-\sum_{i=0}^{r-1} \lambda_{r-i-1}^{0} g^{(i)}(b-)\right\}=0,
\end{aligned}
$$

according to (2.13). The relation (2.14) is proved. Now suppose that $M_{\alpha}(t)$ is an arbitrary function of the form (2.2) with coefficients $\beta_{i}, a_{k \lambda}$, satisfying the boundary conditions (2.3). Then

$$
\begin{aligned}
\int_{\alpha}^{b}\left|M_{\alpha}^{*}(t)\right|^{p} d t & =\int_{a}^{b}\left|M_{\alpha}^{*}(t)\right|^{p-1} M_{\alpha}^{*}(t) \operatorname{sign} M_{\alpha}^{*}(t) d t \\
& =\int_{\alpha}^{b}\left|M_{\alpha}^{*}(t)\right|^{p-1}\left[M_{\alpha}^{*}(t)-M_{\alpha}(t)+M_{\alpha}(t)\right] \operatorname{sign} M_{\alpha}^{*}(t) d t \\
& =\int_{a}^{b}\left|M_{\alpha}^{*}(t)\right|^{p-1} M_{\alpha}(t) \operatorname{sign} M_{\alpha}^{*}(t) d t \\
& <\int_{a}^{b}\left|M_{\alpha}^{*}(t)\right|^{p-1}\left|M_{\alpha}(t)\right| d t
\end{aligned}
$$

Applying Hölder's inequality, we get

$$
\int_{a}^{b}\left|M_{\alpha}^{*}(t)\right|^{p} d t<\left(\int_{a}^{b}\left|M_{\alpha}^{*}(t)\right|^{(p-1) q} d t\right)^{1 / q}\left\|M_{\alpha}\right\|_{p}
$$

Therefore, $\left\|M_{\alpha}^{*}\right\|_{p} \leqslant\left\|M_{\alpha}\right\|_{p}$, which was to be shown. The theorem is proved.

We conclude from Theorem 1 that the best coefficients $\left\{a_{k \lambda}, k=2, \ldots, n\right.$, $\left.\lambda=0, \ldots, \nu_{k}-1\right\}$ are the unique solution of the system (2.8). The rest of them $a_{1 \lambda}, \lambda=0, \ldots, \nu_{1}-1$, can be found by (2.5). 
Let $\omega$ be a $(b-a)$-periodic, integrable in $[a, b]$, function which is positive almost everywhere in $[a, b]$. We shall refer to a function $\omega$ obeying the above requirements as being a weight function.

Definition 3. We call the function

$$
S(x)=\sum_{i=0}^{r-1}\left(a_{i} / i !\right) x^{i}+\int_{a}^{b} \frac{(x-t)_{+}^{r-1}}{(r-1) !} \omega(t) g(t) d t
$$

a $(b-a)$-periodic natural spline function of degree $2 r-1$ with a weight $\omega$ and knots $\mathbf{x}$ if

$$
S^{(j)}(a)=S^{(j)}(b), \quad j=0, \ldots, r-1,
$$

and $g$ is a $(b-a)$-periodic spline of degree $r-1$ with knots $\mathbf{x}$.

Denote by $\Re_{2 r-1}(\omega ; \mathbf{x})$ the set of all such splines. As an immediate consequence of (2.6) and (2.14) we get

Corollary 1. Let $1<p<\infty, L=L_{\alpha}$ and $\alpha \in[0,1]$. Suppose that the coefficients $\left\{a_{k \lambda}\right\}$ are best for the nodes $\mathbf{x}$. Then the rule (1.1) is exact for every $S \in \Re_{2 r-1}(\omega ; \mathbf{x})$ with $\omega(t)=\left|M_{\alpha}(\mathbf{x} ; t)\right|^{p-2}$.

Thus, if we want $L_{\alpha}(f)$ for some particular $f$, an alternative procedure is to operate with $L_{\alpha}$ on the natural spline $S_{f}$ with weight $\left|M_{\alpha}(\mathbf{x} ; t)\right|^{p-2}$ that interpolates $f$ at $\mathbf{x}$. In the next theorem we show that such an interpolating spline exists.

THEOREM 2. Suppose that the weight $\omega$ and the nodes $\mathbf{x}\left(\right.$ with $\left.\nu_{1}+\cdots+\nu_{n}>1\right)$ are given. Then, for every function $f \in \tilde{C}^{r-1}[a, b]$ there exists a unique spline $S \in \mathfrak{N}_{2 r-1}(\omega ; \mathbf{x})$ which satisfies the interpolation conditions

$$
S^{(\lambda)}\left(x_{k}\right)=f^{(\lambda)}\left(x_{k}\right), \quad k=1, \ldots, n, \lambda=0, \ldots, \nu_{k}-1 .
$$

Proof. Evidently, the conditions (2.17), together with (2.13) and (2.16), form a linear system of $\nu_{2}+\cdots+\nu_{n}+2 r$ equations with $\nu_{2}+\cdots+\nu_{n}+2 r$ unknowns: $\left\{a_{i}\right\}_{0}^{r-1},\left\{\gamma_{i}\right\}_{0}^{r-1},\left\{c_{k \lambda}, k=2, \ldots, n, \lambda=0, \ldots, \nu_{k}-1\right\}$. Denote by $\Delta(\omega ; \mathbf{x})$ the determinant of this system of equations ordered as follows: (2.16), (2.17), (2.13). The theorem will be proved if it can be shown that $\Delta(\omega ; \mathbf{x}) \neq 0$ or, which is equivalent, that the corresponding homogeneous system has only a trivial solution. To prove this, let $S_{0} \in \Re_{2 r-1}(\omega ; \mathbf{x})$ be a spline which satisfies the homogeneous system. Consider the integral

$$
\sigma:=\int_{0}^{b} \omega(t) g_{0}^{2}(t) d t=\int_{a}^{b} g_{0}(t) S_{0}^{(r)}(t) d t
$$

where $g_{0}$ is the spline in the representation (2.15) of $S_{0}$. Integrating by parts, we get

$$
\begin{aligned}
\sigma= & \sum_{j=0}^{r-1}(-1)^{r-1-j} g \delta^{(r-1-j)}(b-) S_{0}^{(j)}(b)-\sum_{j=0}^{r-1}(-1)^{r-1-j} g \delta^{(r-1-j)}(a+) S_{0}^{(j)}(a) \\
& +\sum_{k=2}^{n} \sum_{\lambda=0}^{\nu_{k}-1}(-1)^{r-1-\lambda} S_{0}^{(\lambda)}\left(x_{k}\right)\left[g_{0}^{(r-1-\lambda)}\left(x_{k}-\right)-g \delta^{(r-1-\lambda)}\left(x_{k}+\right)\right] .
\end{aligned}
$$

Since $S_{0}$ satisfies the homogeneous system we conclude that $\sigma=0$. This implies $S_{0}^{(r)}(t) \equiv 0$ and, consequently, $S_{0}$ is a polynomial of degree $r-1$. Then, the periodicity of $S_{0}$ yields $S_{0}(t)=$ const. Finally, $S_{0}(t)$ vanishes at least at one point $\left(\nu_{1}+\cdots+\nu_{n} \geqslant 1\right)$, hence $S_{0}(t) \equiv 0$. The theorem is proved. 
As we have already mentioned, Theorem 2 is equivalent to the following

Proposition 1. The determinant $\Delta(\omega ; \mathbf{x})$ is nonzero for every weight function $\omega$ and nodes $\mathbf{x}$.

Now denote by $J(x)$ the Jacobian of the system (2.8) with respect to the coefficients $\left\{a_{i}\right\},\left\{\beta_{i}\right\},\left\{a_{k \lambda}\right\}$ at the solution of (2.8). Our next task is to prove that $J(\mathbf{x})$ is nonsingular. Indeed, careful computation of the elements of $J(\mathbf{x})$ shows that the elements from the first $r+\nu_{1}+\cdots+\nu_{n}$ rows of $J(x)$ are equal to the corresponding ones of $\Delta\left(\left|M_{\alpha}(\mathbf{x} ; t)\right|^{p-2} ; \mathbf{x}\right)$ multiplied by $p-1$; the elements of the last $r-\nu_{1}$ rows of $J$ and $\Delta$ coincide. Therefore,

$$
\operatorname{det} J(\mathbf{x})=(p-1)^{r+\nu_{1}+\cdots+\nu_{n}} \Delta\left(\left|M_{\alpha}(\mathbf{x} ; t)\right|^{p-2} ; \mathbf{x}\right) .
$$

It can be shown (see Appendix, Corollary 3) that the function $\left|M_{\alpha}(\mathbf{x} ; t)\right|^{p-2}$ is integrable on $[a, b]$ if $1<p<\infty$. Thus, we have proved

COROllaRY 2. The Jacobian $J(\mathbf{x})$ is nonsingular for every system of nodes $\mathbf{x}$ of the form (2.1).

Finally, we recall an existence theorem [5, Theorem 2] which will be needed in the sequel.

THEOREM A. Let $\left\{\nu_{k}\right\}_{1}^{n}$ be arbitrary fixed natural numbers satisfying the inequalities $1<\nu_{k}<r, k=1, \ldots, n, \nu_{1}+\cdots+\nu_{n}>1, r>1$. Suppose that $1<q<\infty$. Then there exists a system $\mathbf{x}$ of optimal nodes of type $\left(\nu_{1}, \ldots, \nu_{n}\right)$ in the class $\tilde{W}_{q}^{r}[a, b]$. Moreover, the best coefficients $\left\{a_{k \lambda}\right\}$ for the optimal nodes satisfy the conditions

$$
\begin{cases}a_{k \lambda}>0, \lambda=0,2, \ldots, \nu_{k}-1 & \text { if } \nu_{k} \text { is odd } \\ a_{k, \nu_{k}-1}=0, a_{k \lambda}>0, \lambda=0,2, \ldots, \nu_{k}-2 & \text { if } \nu_{k} \text { is even }\end{cases}
$$

3. Main Result. We prove in this section our central theorem. The proof is based on the concept of topological degree. We picked up the thought of using topological degree in approximation theory from Barrow [3].

First we recall, for the sake of completeness, some facts from degree theory (see Schwartz [17] or Ortega and Rheinboldt [15]).

Let $D$ be an open bounded set in the Euclidean space $\mathbf{R}^{n}$. Denote by $\bar{D}$ and $\partial D$ the closure and the boundary of $D$, respectively. Let the mapping $\Phi: \bar{D} \rightarrow \mathbf{R}^{n}$ be continuous. Then, if $c \in \mathbf{R}^{n}$ and $c \notin \Phi(\partial D)$, the degree of $\Phi$ with respect to $D$ and $c$ is defined, has an integer value and will be denoted by $\operatorname{deg}(\Phi, D, c)$. The following are some basic properties of the degree:

(i) Suppose that $\Phi \in C^{\prime}(D), c \in \Phi(\partial D)$ and let $\operatorname{det}\left(\Phi^{\prime}(x)\right) \neq 0$ for each $x \in D$ satisfying the equation $\Phi(x)=c$. Then there are a finite number of points $x_{i} \in D$ where $\Phi\left(x_{i}\right)=c$ and $\operatorname{deg}(\Phi, D, c)=\Sigma_{i} \operatorname{sign} \operatorname{det}\left(\Phi^{\prime}\left(x_{i}\right)\right)$.

(ii) If $\operatorname{deg}(\Phi, D, c) \neq 0$, there exists at least one point $x \in D$ for which $\Phi(x)=c$.

(iii) Let $\Phi(\alpha ; x)$ be continuous on $[0,1] \times \bar{D}$ and $\Phi(\alpha ; x) \neq c$ for any $x \in \partial D$, $0<\alpha<1$. Then $\operatorname{deg}(\Phi(\alpha ; \cdot), D, c)$ is constant independently of $\alpha$.

(iv) Let $c \notin \Phi(\partial D), c_{m} \notin \Phi(\partial D) \cup \Phi(z(D)), \quad m=1,2, \ldots$, where $z(D):=$ $\left\{x \in D: \operatorname{det}\left(\Phi^{\prime}(x)\right)=0\right\}$. Suppose that $c_{m} \rightarrow c$ as $m \rightarrow \infty$. Then $\operatorname{deg}(\Phi, D, c)=$ $\operatorname{deg}\left(\Phi, D, c_{m}\right)$ for each sufficiently large $m$. 
Now, let us introduce the notations

$$
\begin{aligned}
\Omega & :=\left\{\bar{x} \in \mathbf{R}^{n-1}: \bar{x}=\left(x_{2}, \ldots, x_{n}\right), a=x_{1}<x_{2}<\cdots<x_{n}<b\right\}, \\
\Omega_{\varepsilon} & :=\left\{\bar{x} \in \Omega: x_{i+1}-x_{i}>\varepsilon, i=1, \ldots, n, x_{n+1}:=b\right\} .
\end{aligned}
$$

Define the mapping $\Phi(\alpha ; \bar{x}): \Omega \rightarrow \mathbf{R}^{n-1}$ by

$$
\Phi(\alpha ; \bar{x}):=\left(b_{2}(\alpha ; \bar{x}), \ldots, b_{n}(\alpha ; \bar{x})\right), \quad \bar{x} \in \Omega,
$$

where we have denoted by $b_{k}(\alpha ; \bar{x})$ the coefficient $a_{k, \nu_{k}-1}$ of the monospline $M_{\alpha}(\mathbf{x} ; t), \mathbf{x}=\left(\begin{array}{c}a, x_{2}, \ldots, x_{n} \\ p_{1}, v_{2}, \ldots, v_{n}\end{array}\right)$.

The basic steps of our reasoning in the proof of the uniqueness of the optimal nodes go through the following lemmas.

LEMmA 1. Let $1<p<\infty, \alpha=0$ and $r>1$. Suppose that the parameters $\left\{a_{i}\right\}$, $\left\{\beta_{i}\right\},\left\{a_{k \lambda}\right\}$ of the function $F_{\alpha}(\mathbf{x} ; t)$ satisfy the system $(2.8)$ for some $\mathbf{x}$. Then the equalities $a_{k, v_{k}-1}=0$ for $k=2, \ldots, n$ imply $M_{0}(\mathbf{x} ; t) \equiv 0$, i.e., the system

$$
a_{k, v_{k}-1}\left(x_{2}, \ldots, x_{n}\right)=0, \quad k=2, \ldots, n,
$$

has a unique solution $x_{k}=y_{k}, k=2, \ldots, n$.

Lemma 2. Let $1<p<\infty$ and $r>1$. Suppose that $\left\{\nu_{k}\right\}_{1}^{n}$ are even multiplicities. Then there exists an $\varepsilon>0$ such that whenever $\Phi(\alpha ; \bar{x})=\overline{0}(\overline{0}:=(0, \ldots, 0) \in$ $\left.\mathbf{R}^{n-1}\right)$ for some $\alpha \in[0,1]$ and $\bar{x} \in\left(x_{2}, \ldots, x_{n}\right) \in \Omega$, then $\bar{x} \in \Omega_{e}$.

LEMMA 3. Let $1<p<\infty$ and $r>1$. Suppose that $\left\{\nu_{k}\right\}_{1}^{n}$ are even multiplicities. Let the points $\bar{y}:=\left(y_{2}, \ldots, y_{n}\right)$ be fixed and $a<y_{2}<\cdots<y_{n}<b$. Suppose that $\varepsilon$ is an arbitrary positive number such that $\bar{y} \in \Omega_{\varepsilon}$. Then

$$
\operatorname{deg}\left(\Phi(0 ; \cdot), \Omega_{e}, \overline{0}\right)=(-1)^{n-1} .
$$

Denote by $\Delta_{j k}(\omega ; \mathbf{x})$ the matrix which is obtained from $\Delta(\omega ; \mathbf{x})$ by deleting the row and the column of numbers $r+\nu_{1}+\cdots+\nu_{j}$ and $2 r+\nu_{2}+\cdots+\nu_{k}$, respectively.

LEMMA 4. Let $1<p<\infty, r>1$. Suppose that $\left\{\nu_{k}\right\}_{1}^{n}$ are even multiplicities satisfying the conditions $1<\nu_{k}<r, k=1, \ldots, n$, and $\omega$ is an arbitrary $(b-a)$ periodic weight. Let $\bar{x}=\left(x_{2}, \ldots, x_{n}\right) \in \Omega$. Then, for fixed $k \in\{2, \ldots, n\}$, the determinants det $\Delta_{j k}(\omega ; \mathbf{x}), j=1, \ldots, n$, are nonzero and have a constant sign, independent of $j$ and $\omega$.

Now we are prepared to prove our central theorem.

THEOREM 3. Let $\left\{\nu_{k}\right\}_{1}^{n}$ be arbitrary fixed natural numbers satisfying the conditions $1<2\left[\left(\nu_{k}+1\right) / 2\right]<r, k=1, \ldots, n, r>1$. Suppose that $1<q<\infty$. Then there exists a unique optimal quadrature formula of type $\left(\nu_{1}, \ldots, \nu_{n}\right)$ in the class $\tilde{W}_{q}^{r}[a, b]$ with fixed node $x_{1}=a$.

Proof. It is clear from Theorem A that the assertion will be proved if we show that the equation

$$
\Phi(1 ; \bar{x})=\overline{0}
$$

has a unique solution for every system $\left\{\nu_{k}\right\}_{1}^{n}$ of even multiplicities. For $r=1$, the optimal nodes can be found even explicitly; see [11]. So, we assume in the sequel that $r>1$. 
Let the number $q$ be fixed in $(1, \infty)$. According to Lemma 2 , there exists an $\varepsilon>0$ such that each solution $\bar{x}$ of the equation $\Phi(\alpha ; \bar{x})=\overline{0}$ is situated in $\Omega_{\varepsilon}$ for any $\alpha \in[0,1]$. It is easily seen that $\Phi(\alpha ; \bar{x})$ is a continuous function of $\alpha$ and $\bar{x}$ in $[0,1] \times \Omega_{e}$. Then, using (iii), we conclude from Lemma 3 that

$$
\operatorname{deg}\left(\Phi(1 ; \cdot), \Omega_{e}, \overline{0}\right)=\operatorname{deg}\left(\Phi(0 ; \cdot), \Omega_{e}, \overline{0}\right)=(-1)^{n-1}
$$

Next, observe that $\Phi(1 ; \cdot) \in C^{\prime}\left(\Omega_{\mathrm{e}}\right)$. Indeed, the coefficients $b_{k}(1 ; \bar{x})$ are solutions of the system (2.8) for $\alpha=1$. Further, the Jacobian $J$ of the system (2.8) is nonsingular, in view of Corollary 2 . Thus, by the implicit function theorem, $b_{k}(1 ; \bar{x})$ is a differentiable function of $\bar{x}$. Moreover,

$$
\frac{\partial b_{k}(1 ; \bar{x})}{\partial x_{j}}=-\frac{\operatorname{det} A_{k j}}{\operatorname{det} J},
$$

where the matrix $A_{k j}$ differs from $J$ only by its $2 r+\nu_{2}+\cdots+\nu_{k}$ th column which consists of partial derivatives with respect to $x_{j}$, but not with respect to $b_{k}$, as it is in $J$. The elements of $A_{k j}$ and $J$ are continuous functions of $\bar{x}$ and $\operatorname{det} J(\bar{x}) \neq$ 0 . Therefore, $\Phi(1 ; \bar{x}) \in C^{\prime}\left(\Omega_{\varepsilon}\right)$. Then the uniqueness of the solution of (3.2) will follow immediately from (i) and (3.3) if we prove that

$$
\text { sign } \operatorname{det}\left(\frac{\partial \Phi(1 ; \bar{x})}{\partial \bar{x}}\right)=(-1)^{n-1}
$$

for each $\bar{x} \in \Omega_{e}$ satisfying (3.2). To this end, we assume that $\bar{x}=\left(x_{2}, \ldots, x_{n}\right)$ satisfies (3.2) and $\bar{x} \in \Omega_{e}$. Denote for simplicity the function $F_{1}(\mathbf{x} ; t)$ (from (2.11)) by $F(t)$. Careful calculation shows (see (3.4)) that

$$
\begin{aligned}
& \frac{\partial b_{k}(1 ; \bar{x})}{\partial x_{j}}=-(-1)^{r} F^{\left(v_{j}\right)}\left(x_{j}\right) \operatorname{det} J_{j k} / \operatorname{det} J \text { for } k \neq j, \\
& \frac{\partial b_{j}(1 ; \bar{x})}{\partial x_{j}}=-(-1)^{r} F^{\left(v_{j}\right)}\left(x_{j}\right) \operatorname{det} J_{j j} / \operatorname{det} J-a_{j, v_{j}-2},
\end{aligned}
$$

where the the matrix $J_{j k}$ is obtained from $J$ by deleting the column and the row of numbers $2 r+\nu_{2}+\cdots+\nu_{k}$ and $r+\nu_{1}+\cdots+\nu_{j}$, respectively.

Clearly,

$$
\operatorname{det} J_{j k}=(p-1)^{r+\nu_{1}+\cdots+\nu_{n}-1} \operatorname{det} \Delta_{j k}\left(\left|M_{1}(\mathbf{x} ; t)\right|^{p-2} ; \mathbf{x}\right) \text {. }
$$

Therefore, in view of Lemma 4 , det $J_{j k}$ has a constant sign for $k \in\{2, \ldots, n\}$, $j=1, \ldots, n$. Now, consider the values $F^{\left(y_{j}\right)}\left(x_{j}\right), j=1, \ldots, n$. It follows from the construction of $F$ that it has $\nu_{1}+\cdots+\nu_{n}$ zeros. Consequently, by Rolle's theorem, $M_{1}(\mathbf{x} ; t)$ has at least $\nu_{1}+\cdots+\nu_{n}$ zeros in $[a, b)$. Further, the assumption $\Phi(1 ; \bar{x})=\overline{0}$ shows that the knots $x_{2}, \ldots, x_{n}$ of $M_{1}(\mathbf{x} ; t)$ have odd multiplicities. Then, according to Lemma 4 from [5], the coefficients $\left\{a_{k \lambda}\right\}$ of $M_{1}(\mathbf{x} ; t)$ satisfy the inequality

$$
a_{k, v_{k}-2}>0, \quad k=2, \ldots, n .
$$

It is easily seen that the function $F(t)$ has no other zeros except $\left\{x_{k}\right\}_{1}^{n}$ of multiplicities $\left\{\nu_{k}\right\}_{1}^{n}$ (Otherwise $M_{1}(\mathbf{x} ; t)$ would have more than $\nu_{1}+\cdots+\nu_{n}$ zeros, a contradiction to a known result of Micchelli [13, Proposition 1]; see Lemma 8b of the Appendix.) Since $\left\{\nu_{k}\right\}_{1}^{n}$ are even, $F^{\left(\nu_{k}\right)}\left(x_{k}\right)$, and consequently $(-1)^{r} F^{\left(\nu_{k}\right)}\left(x_{k}\right)$ has 
a constant sign for $k=1, \ldots, n$. So, we proved that the quantities

$$
\gamma_{k j}:=(-1)^{r} F^{\left(v_{k}\right)}\left(x_{k}\right) \operatorname{det} J_{j k} / \operatorname{det} J
$$

have a constant sign for $k \in\{2, \ldots, n\}, j=1, \ldots, n$.

Next observe that $M_{1}^{\prime}(\mathbf{x} ; t)$ is a periodic spline function of degree $r-1$ with knots $\left\{x_{k}\right\}_{1}^{n}$ of multiplicities $\left\{\nu_{k}\right\}_{1}^{n}$, respectively, since $\Phi(1 ; \bar{x})=\overline{0}$ (which implies $a_{k, v_{k}-1}=0, k=1, \ldots, n$, as can be seen in the proof of Lemma 1, Appendix). Therefore $F^{\prime} \in \mathscr{T}_{2 r-1}(\omega ; \mathbf{x})$ where $\omega(t)=\left|M_{1}(\mathbf{x} ; t)\right|^{p-2}$ and $F^{\prime}$ can be presented (according to Theorem 2) in the form

$$
F^{\prime}(x)=F^{\left(v_{1}\right)}\left(x_{1}\right) S_{1}(x)+\cdots+F^{\left(v_{n}\right)}\left(x_{n}\right) S_{n}(x),
$$

where $S_{i} \in \mathscr{T}_{2 r-1}(\omega ; \mathbf{x})$ and

$$
\begin{aligned}
S_{i}^{(j)}(a) & =S_{i}^{(j)}(b), \quad j=0, \ldots, r-1, \\
S_{i}^{(\lambda)}\left(x_{k}\right) & =0, \quad k \neq i, \lambda=0, \ldots, \nu_{k}-1, \\
S_{i}^{(\lambda)}\left(x_{i}\right) & =0, \quad \lambda=0, \ldots, \nu_{i}-2, \\
S_{i}^{\left(\nu_{i}-1\right)}\left(x_{i}\right) & =1, \\
g^{(j)}(a+) & =g^{(j)}(b-), \quad j=0, \ldots, r-\nu_{1}-1 .
\end{aligned}
$$

Here $g$ is the spline in the representation (2.15) of $S_{i}$. Now, following an idea of [8], we compare the coefficients of the basic functions on both sides of (3.8) and obtain

$$
-(p-1) a_{k, v_{k}-2}=\sum_{j=1}^{n} F^{\left(v_{j}\right)}\left(x_{j}\right)\left\{(-1)^{r}(p-1) \operatorname{det} J_{j k} / \operatorname{det} J\right\} .
$$

Therefore,

$$
a_{k, \nu_{k}-2}=\sum_{j=1}^{n} \gamma_{k j}, \quad k=2, \ldots, n
$$

Since $\gamma_{k j}$ has a constant sign, we deduce from (3.7) that

$$
\gamma_{k j}>0, \quad k=2, \ldots, n, j=1, \ldots, n \text {. }
$$

Therefore, $a_{k, v_{k}-2}-\left(\gamma_{k 2}+\cdots+\gamma_{k n}\right)>0$. This inequality shows that the determinant

$$
\operatorname{det}\left(\frac{\partial \Phi(1 ; \bar{x})}{\partial \bar{x}}\right)=\left|\begin{array}{cccc}
\gamma_{22}-a_{2, \nu_{2}-2} & \gamma_{23} & \ldots & \gamma_{2 n} \\
\gamma_{32} & \gamma_{33}-a_{3, \nu_{3}-2} & \ldots & \gamma_{3 n} \\
\ldots \ldots \ldots \ldots & \ldots \ldots \ldots \ldots \ldots & \ldots \ldots \\
\gamma_{n 2} & \gamma_{n 3} & \ldots & \gamma_{n n}-a_{n, \nu_{n}-2}
\end{array}\right|
$$

has a dominant main diagonal, i.e., the corresponding matrix is strictly diagonally dominant. Moreover (see [4]),

$$
\text { sign det }\left(\frac{\partial \Phi(1 ; \bar{x})}{\partial \bar{x}}\right)=\operatorname{sign} \prod_{k=2}^{n}\left(\gamma_{k k}-a_{k, v_{k}-2}\right)=(-1)^{n-1}
$$

The relation (3.5) is proved. This completes the proof of our theorem.

The next proposition is a simple consequence of the previous theorem. 
TheOREM 4. Let $\nu_{1}=\cdots=\nu_{n}=\rho$ and $1<2[(\rho+1) / 2]<r$. Then the equidistant nodes

$$
x_{k}=a+\frac{k-1}{n}(b-a), \quad k=1, \ldots, n,
$$

are optimal of type $\left(\nu_{1}, \ldots, \nu_{n}\right)$ in the class $\tilde{W}_{q}^{r}[a, b]$ for $1<q<\infty$. Moreover, the best coefficients $\left\{a_{k \lambda}\right\}$ for these nodes satisfy the conditions

$$
\begin{aligned}
& a_{1 \lambda}=\cdots=a_{n \lambda}=0 \quad \text { if } \lambda \text { is odd, } \\
& a_{1 \lambda}=\cdots=a_{n \lambda}>0 \text { if } \lambda \text { is even, } \\
& a_{10}=\cdots=a_{n 0}=(b-a) / n .
\end{aligned}
$$

The optimal nodes are unique in the case $1<q<\infty$.

Proof. Suppose that $1<q<\infty$ and $\nu_{1}=\cdots=\nu_{n}=\rho$. It follows from the uniqueness of the optimal nodes that they must be equidistant.

Set

$$
\mathbf{x}=\left(\begin{array}{c}
x_{1} \cdots x_{n} \\
\rho \cdots \rho
\end{array}\right), \quad \omega(t)=\left|M_{1}(\mathbf{x} ; t)\right|^{p-2}
$$

where $1 / p+1 / q=1$. Denote by $\varphi_{i j}$ the unique function from the set $\Re_{2 r-1}(\omega ; \mathbf{x})$ which satisfies the interpolation conditions

$$
\varphi_{i j}^{(\lambda)}\left(x_{k}\right)=\delta_{i k} \delta_{\lambda j}
$$

$\delta_{i j}$ being the Kronecker symbol. On the basis of Corollary $1, a_{k \lambda}=\int_{a}^{b} \varphi_{k \lambda}(t) d t$. Since the function $\varphi_{k \lambda}(t)$ can be obtained from $\varphi_{1 \lambda}(t)$ by translation, we conclude that

$$
a_{1 \lambda}=\cdots=a_{n \lambda} \text { for } \lambda=0, \ldots, \rho-1 .
$$

Further, it is easily seen that the quadrature formula

$$
\int_{a}^{b} f(t) d t \approx \sum_{\lambda=0}^{\nu_{1}-1}(-1)^{\lambda} a_{1 \lambda} f^{(\lambda)}\left(x_{1}\right)+\sum_{k=2}^{n} \sum_{\lambda=0}^{\nu_{k}-1}(-1)^{\lambda} a_{n+2-k, \lambda} f^{(\lambda)}\left(x_{k}\right)
$$

has the same error in $\tilde{W}_{q}^{r}[a, b]$ as the optimal quadrature. Then, it follows from Theorem 3 that

$$
(-1)^{\lambda} a_{1 \lambda}=a_{1 \lambda}, \quad \lambda=0, \ldots, \nu_{1}-1 .
$$

This, coupled with (3.10), gives $a_{k \lambda}=0, k=1, \ldots, n$, for odd $\lambda$.

The value of $a_{k 0}$ is calculated using the fact that the optimal quadrature formula is exact for $f(t) \equiv 1$.

The optimality of the equidistant nodes for $q=1, \infty$ follows as a limiting case. The theorem is proved.

Note that a particular case of Theorem 4 (for $\rho=r-2$ ) was studied in [12].

Using the correspondence between monosplines and quadrature formulae, we can restate our main result in the following form.

THEOREM 5. Let $\left\{\nu_{k}\right\}_{1}^{n}$ be arbitrary fixed natural numbers satisfying the conditions $1<2\left[\left(\nu_{k}+1\right) / 2\right]<r, k=1, \ldots, n$. Suppose that $1<p<\infty$. Then there exists $a$ unique (up to translation) $(b-a)$-periodic monospline of least $L_{p}$ deviation in $[a, b]$ of degree $r$ with $n$ distinct free knots $\left\{x_{k}\right\}_{1}^{n}$ of fixed multiplicities $\left\{\nu_{k}\right\}_{1}^{n}$. 
An analogous proposition to Theorem 5 was proved in [6] for monosplines with free ends, i.e., without boundary conditions. The first uniqueness results for monosplines of least $L_{p}$ deviation are due to Johnson [9] (for $p=\infty, \nu_{1}=\cdots=$ $\nu_{n}=1$, free ends), Jetter and Lange [8] (for $p=2, \nu_{1}=\cdots=\nu_{n}=1$, free ends) and Žensykbaev [21] (for $1<p<\infty, \nu_{1}=\cdots=\nu_{n}=1$, under the boundary conditions $\left.M^{(j)}(a)=M^{(j)}(b)=0, j=0, \ldots, r-1\right)$. Recently Barrar and Loeb [2] considered the case $p=\infty$ with arbitrary fixed $\left\{\nu_{k}\right\}_{1}^{n}, 1<\nu_{k}<r, k=1, \ldots, n$, and free ends.

Remark 2. We must note that the proofs of Lemma 5 and Lemma 7 of [6] are not complete. However, one can apply with obvious modifications the reasoning used in Lemma 1 and Lemma 2 of the present paper in order to complete the proofs of the lemmas mentioned.

\section{Appendix}

First we shall recall some known facts about spline functions and derive some new properties of the function $M_{\alpha}(\mathbf{x} ; t)$ which we require in our study.

For the sake of convenience we extend the definition of $M_{\alpha}(\mathbf{x} ; t)$ by

$$
M_{\alpha}(\mathbf{x} ; t):=M_{\alpha}\left([\mathbf{x}]_{r} ; t\right)
$$

for each $\mathbf{x}=\left(\begin{array}{c}x_{1} \cdots x_{n} \\ m_{1} \cdots m_{n}\end{array}\right)$, where $[\mathbf{x}]_{r}:=\left(\begin{array}{c}x_{1} \cdots x_{n} \\ \mu_{1} \cdots \mu_{n}\end{array}\right)$, and $\mu_{k}=\min \left(r, m_{k}\right), k=1, \ldots, n$.

LEMMA 5. Let $1<p<\infty$,

$$
\mathbf{x}_{0}=\left(\begin{array}{l}
\xi_{1} \cdots \xi_{j} \\
\rho_{1} \cdots \rho_{j}
\end{array}\right), \quad \mathbf{x}_{m}=\left(\begin{array}{r}
x_{m 1} \cdots x_{m n} \\
\nu_{1} \cdots \nu_{n}
\end{array}\right), \quad m=1,2, \ldots
$$

Suppose that $\mathbf{x}_{m} \rightarrow \mathbf{x}_{0}$ in $\mathbf{R}^{N}, N=\nu_{1}+\cdots+\nu_{n}, \alpha_{m} \rightarrow \alpha_{0}, 0<\alpha_{m}<1, m=$ $1,2, \ldots$, as $m \rightarrow \infty$. Then $M_{\alpha_{m}}\left(\mathbf{x}_{m} ; t\right)$ converges uniformly to $M_{\alpha_{0}}\left(\mathbf{x}_{0} ; t\right)$ on each compact subset of $[a, b)$ which does not contain breakpoints of $M_{\alpha_{0}}\left(\mathrm{x}_{0} ; t\right)$.

This lemma was proved in [5] for $\alpha_{m}=1, m=1,2, \ldots$ The proof in the present case is similar. We omit it.

LEMMA 6a. Let the multiplicities $\left\{\nu_{k}\right\}_{1}^{n}$ be odd. Given $[a, b]$ there exists a constant $C>0$ such that whenever $M(t)$ is a function of the form

$$
\begin{aligned}
M(t)= & \alpha \frac{(b-t)^{r}}{r !}+(1-\alpha) \sum_{k=1}^{m} \frac{\left(y_{k}-t\right)_{+}^{r-1}}{(r-1) !}+\sum_{i=0}^{r-1} \beta_{i} \frac{(b-t)^{i}}{i !} \\
& -\sum_{k=1}^{n} \sum_{\lambda=0}^{\nu_{k}-1} a_{k \lambda} \frac{\left(x_{k}-t\right)_{+}^{r-\lambda-1}}{(r-\lambda-1) !}
\end{aligned}
$$

with $0<\alpha<1, x_{1}<\cdots<x_{n}$ and $M$ has $r+\sum_{i=1}^{n}\left(\nu_{i}+1\right)$ distinct zeros in $[a, b]$, then

$$
\begin{aligned}
\left|\beta_{i}\right|<C, & i=0, \ldots, r-1, \\
\left|a_{k \lambda}\right|<C, & k=1, \ldots, n, \lambda=0, \ldots, \nu_{k}-1 .
\end{aligned}
$$

The lemma was proved in [13] for $\alpha=1$. A similar proof can be given for this setting. 
LEMMA 6b. Let the multiplicities $\left\{\nu_{k}\right\}_{1}^{n}$ be odd. Given $[a, b]$, there exists a constant $C>0$ such that, whenever $M_{\alpha}(t)$ is a function of the form (2.2) satisfying (2.3) with $0<\alpha<1$ and $M_{\alpha}$ has $\sum_{i=1}^{n}\left(\nu_{i}+1\right)$ distinct zeros in $[a, b)$, then $M_{\alpha}$ has coefficients bounded by $C$.

Proof. If some $\nu_{i}$ is equal to $r$, we apply Lemma $6 a$ to $M_{\alpha}(t)$ considered on the interval $\left[x_{i}, x_{i}+b-a\right]$. Suppose that $\max _{k} \nu_{k}=\nu_{i}<r$. Then $M_{\alpha}^{\left(r-r_{i}\right)}(t)$ has $\sum_{i=1}^{n}\left(\nu_{i}+1\right)$ distinct zeros in $\left[x_{i}, x_{i}+b-a\right)$ and, according to Lemma 6a, $M_{\alpha}^{\left(r-v_{i}\right)}(t)$ has bounded coefficients. It is easy to see that $\beta_{0}, \ldots, \beta_{r-v_{1}-1}$ are also bounded. For example

$$
\beta_{r-v_{i}-1}+\int_{a}^{\tau} M_{\alpha}^{\left(r-v_{i}\right)}(t) d t=0
$$

for some $\tau \in[a, b)$. Therefore, $\left|\beta_{r-v_{t}-1}\right|<(b-a)\left\|M_{\alpha}^{\left(r-v_{1}\right)}\right\|_{\infty}$. The latter norm is bounded since the coefficients of $M_{\alpha}^{\left(r-v_{i}\right)}(t)$ are bounded. The lemma is proved.

Denote by $Z(s ; \mathbb{Q})$ the number of zeros of the spline $s$ in the set $\mathbb{Q}$ where the multiplicities are counted as in Schumaker [16]. The following is a result of Schumaker [16].

LEMMA 7. Let $s$ be $a(b-a)$-periodic spline function of degree $r-1$ with knots $\left\{\xi_{i}\right\}_{1}^{m}, a<\xi_{1}<\cdots<\xi_{m}<b$, of multiplicities $\left\{\mu_{i}\right\}_{1}^{m}$, respectively. Suppose that $s$ does not vanish identically. Then

$$
\begin{aligned}
Z(s ;[a, b)) & \leqslant K-1 & & \text { if } K \text { is odd, } \\
& \leqslant K & & \text { if } K \text { is even, }
\end{aligned}
$$

where $K=\mu_{1}+\cdots+\mu_{m}$.

The next lemma was proved in [6].

Lemma 8a. Let $0<\alpha<1$. Suppose that $M(t)$ is a function of the form (1). Then

$$
Z(M ;[a, b])<r+\sum_{k=1}^{n}\left(\nu_{k}+\sigma_{k}\right),
$$

where $\sigma_{k}=1$, if $\nu_{k}$ is odd, and zero otherwise.

Lemma $8 \mathrm{~b}$. Let $0<\alpha<1$. Suppose that $M_{\alpha}(t)$ is defined by (2.2) and (2.3). Then

$$
Z\left(M_{\alpha} ;[a, b)\right)<\sum_{k=1}^{n}\left(\nu_{k}+\sigma_{k}\right) .
$$

The assertion follows from Micchelli's result [13, Proposition 1] as in the previous lemma.

COROllary 3. Suppose that $\mathbf{x}$ is a given system of nodes with even multiplicities $\nu_{1}, \ldots, \nu_{n}$. Let $1<p<\infty$ and $0<\alpha<1$. Then the function $\left|M_{\alpha}(\mathbf{x} ; t)\right|^{p-2}$ is integrable in $[a, b]$.

Proof. The assertion is evident for $p>2$. Assume that $1<p<2$. Then it is sufficient to show that $M_{\alpha}(\mathbf{x} ; t)$ has only simple zeros in $[a, b)$. To this end, recall that the function $F_{\alpha}(\mathbf{x} ; t)$ (see (2.11)) satisfies (2.8). Hence, $F_{\alpha}(\mathbf{x} ; t)$ has at least $N:=\nu_{1}+\cdots+\nu_{n}$ zeros in $[a, b)$. By Rolle's theorem, $F_{\alpha}^{(r)}(\mathbf{x} ; t)$ and, consequently, $M_{\alpha}(\mathbf{x} ; t)$ must have $N$ sign changes in $[a, b)$. This means that $M_{\alpha}(\mathbf{x} ; t)$ 
has $N$ distinct zeros in $[a, b)$. On the other hand, according to Lemma $8 \mathrm{~b}$, $Z\left(M_{\alpha}(\mathbf{x} ; \cdot) ;[a, b)\right)<N$. Therefore, all zeros of $M_{\alpha}(\mathbf{x} ; t)$ are simple. The claim is proved.

Proof of Lemma 1. Let us assume the contrary: $a_{k, p_{k}-1}=0$ for $k=2, \ldots, n$ but $M_{0}(\mathbf{x} ; t)$ is not identically zero. Then, clearly, $F_{0}(\mathbf{x} ; t)$ is not zero, either. We shall show that $F_{0}(\mathbf{x} ; t)$ cannot vanish even on some subinterval of $[a, b)$. Indeed, suppose that $F_{0}(\mathbf{x} ; t)=0$ for every $t$ from a subinterval of $[a, b)$. Then there is a subinterval $\left(t_{1}, t_{2}\right)$ such that $F_{0}(\mathbf{x} ; t) \neq 0$ almost everywhere in $\left(t_{1}, t_{2}\right)$ and, $F_{0}(\mathbf{x} ; t)$ $=0$ if $t \in\left[t_{1}-\varepsilon, t_{1}\right] \cup\left[t_{2}, t_{2}+\varepsilon\right]$ for some $\varepsilon>0$. (Remember that we are thinking of $[a, b)$ as a circle.) Let $y_{l}<t_{1}<y_{l+1}<\cdots<y_{l+j}<t_{2}<y_{l+j+1}, x_{i}<$ $t_{1}<x_{i+1}<\cdots<x_{i+m}<t_{2} \leqslant x_{i+m+1}$. Then $F_{0}(\mathbf{x} ; t)$ has $2 r+N_{i m}, N_{i m}:=\nu_{i+1}$ $+\cdots+\nu_{i+m+1}$, zeros at least in $\left[t_{1}, t_{2}\right]$. Consequently, by Rolle's theorem, $M_{0}(\mathbf{x} ; t)$ has $r+N_{i m}$ sign changes at least in $\left(t_{1}, t_{2}\right)$. Moreover, $M_{0}\left(\mathbf{x} ; t_{1}\right)=0$ or $M_{0}\left(\mathbf{x} ; t_{2}\right)=0$ since $M_{0}(\mathbf{x} ; t)=0$ for $t \in\left[t_{1}-\varepsilon, t_{1}\right] \cup\left[t_{2}, t_{2}+\varepsilon\right]$ and $M_{0}(\mathbf{x} ; t)$ is continuous at least at one of the points $t_{1}, t_{2}$ because $t_{1}, t_{2} \in$ $\left\{x_{1}, \ldots, x_{n}, y_{2}, \ldots, y_{n}\right\}$ and the multiplicities of all these knots, without $x_{1}$, are less than $r$, according to the assumption: $1<v_{k} \leqslant r, k=1, \ldots, n, a_{k, \nu_{k}-1}=0$ for $k=2, \ldots, n$ and $r>1$. Therefore $M_{0}^{\prime}(\mathbf{x} ; t)$ must have at least $r+N_{i m}$ sign changes in $\left(t_{1}, t_{2}\right)$. Then the function

$$
\alpha\left\{\frac{(b-t)^{r-1}}{(r-1) !}-\sum_{k=l+1}^{l+j} \frac{\left(y_{k}-t\right)_{+}^{r-2}}{(r-2) !}\right\}+M_{0}^{\prime}(\mathbf{x} ; t)
$$

must have, for small $\alpha$, at least $r+N_{i m}$ zeros in $\left(t_{1}, t_{2}\right)$, which contradicts Lemma $8 \mathrm{a}$. Therefore $F_{0}(\mathbf{x} ; t)$ has only isolated zeros.

Next we prove that the assumption $a_{k, v_{k}-1}=0$ for $k=2, \ldots, n$, implies $a_{1, v_{1}-1}$ $=0$. For this, we consider the periodic function $F_{0}(\mathbf{x} ; t)$ on an interval $[A, B]$ of length $b-a$ such that $A<x_{1}<\cdots<x_{n}<B, A<y_{2}<\cdots<y_{n}<B$. Then $F_{0}(\mathbf{x} ; t)$ can be represented on $[A, B]$ (see the analysis in the proof of Theorem 1 ) in the form

$$
\begin{aligned}
F_{0}(x)= & F_{0}(\mathbf{x} ; x)=\frac{1}{p} \sum_{j=0}^{r-1}(-1)^{j+1} \lambda_{j} \frac{(x-A)^{r-1-j}}{(r-1-j) !} \\
& +\int_{A}^{B} \frac{(x-t)_{+}^{r-1}}{(r-1) !}\left|M_{0}(t)\right|^{p-2} M_{0}(t) d t
\end{aligned}
$$

where

$$
\begin{aligned}
M_{0}(t)= & M_{0}(\mathbf{x} ; t)=\sum_{k=2}^{n} \frac{\left(y_{k}-t\right)_{+}^{r-1}}{(r-1) !}+P(t) \\
& -\sum_{k=1}^{n} \sum_{\lambda=0}^{\nu_{k}-1} a_{k \lambda} \frac{\left(x_{k}-t\right)_{+}^{r-\lambda-1}}{(r-\lambda-1) !},
\end{aligned}
$$

with some $P \in \pi_{r-1}$, and $\left\{\lambda_{j}\right\}_{0}^{r-1}$ are Lagrange multipliers for the extremal problem

$$
\Psi(M) \rightarrow \text { minimum }
$$


over all splines $M$ of the form (2) with free coefficients. Here

$$
\Psi(M):=\int_{A}^{B}|M(t)|^{p} d t+\sum_{j=0}^{r-1} \lambda_{j}\left[M^{(j)}(B)-M^{(j)}(A)\right] .
$$

By the definition, $M_{0}$ is the unique solution of the system

$$
\left\{\begin{array}{l}
F_{0}^{(j)}(A)=F_{0}^{(j)}(B), \quad j=0, \ldots, r-1, \\
F_{0}^{(\lambda)}\left(x_{k}\right)=0, \quad k=1, \ldots, n, \lambda=0, \ldots, \nu_{k}-1, \\
M_{0}^{(j)}(A+)=M_{0}^{(j)}(B-), \quad j=0, \ldots, r-1 .
\end{array}\right.
$$

Since

$$
\frac{1}{p} \frac{\partial \Psi\left(M_{0}\right)}{\partial x_{k}}=\sum_{\lambda=1}^{\nu_{k}} a_{k, \lambda-1} F_{0}^{(\lambda)}\left(x_{k}\right)
$$

the assumption $a_{k, \nu_{k}-1}=0$, together with (4), yields $\partial \Psi\left(M_{0}\right) / \partial x_{k}=0$ for $k=$ $2, \ldots, n$. We claim that $\partial \Psi\left(M_{0}\right) / \partial x_{1}=0$, too. Indeed, let us set $\Psi\left(\xi_{1}, \ldots, \xi_{n}\right):=\Psi\left(M_{0}(\xi ; \cdot)\right), \xi=\left(\begin{array}{c}\xi_{1} \cdots \xi_{n} \\ \nu_{1} \cdots v_{n}\end{array}\right)$. Then it follows from the periodicity of $M_{0}(\mathbf{x} ; t)$ that

$$
\begin{aligned}
\Psi\left(x_{1}+h, x_{2}, \ldots, x_{n}\right)=\Psi\left(x_{1}, x_{2}-h, \ldots, x_{n}-h\right) \\
=\Psi\left(x_{1}, \ldots, x_{n}\right)-\frac{\partial \Psi\left(M_{0}\right)}{\partial x_{2}} \cdot h-\cdots-\frac{\partial \Psi\left(M_{0}\right)}{\partial x_{n}} \cdot h+o(h) \\
=\Psi\left(x_{1}, \ldots, x_{n}\right)+o(h) .
\end{aligned}
$$

Therefore,

$$
\frac{\partial \Psi\left(M_{0}\right)}{\partial x_{1}}=\lim _{h \rightarrow 0}\left\{\Psi\left(x_{1}+h, x_{2}, \ldots, x_{n}\right)-\Psi\left(x_{1}, \ldots, x_{n}\right)\right\} / h=0,
$$

which was to be shown. Now return to (5). It follows from (4) that

$$
a_{1, \nu_{1}-1} F_{0}^{\left(\nu_{1}\right)}\left(x_{1}\right)=0 \text {. }
$$

Suppose that $F_{0}^{\left(\nu_{1}\right)}\left(x_{1}\right)=0$. Then we conclude, on the basis of Rolle's theorem, that $M_{0}(\mathbf{x} ; t)$ has at least $\nu_{1}+\cdots+\nu_{n}+1$ zeros in $[a, b)$. On the other hand, in view of Lemma $7, Z\left(M_{0} ;[a, b)\right) \leqslant n-1+\nu_{1}+\nu_{2}-1+\cdots+\nu_{n}-1=\nu_{1}$ $+\cdots+\nu_{n}$ or $M_{0}(\mathbf{x} ; t) \equiv 0$. We obtained a contradiction. Therefore, $F_{0}^{\left(\nu_{1}\right)}\left(x_{1}\right) \neq$ 0 . Then the relation (6) implies $a_{1, \nu_{1}-1}=0$. Since $F_{0}(t)$ has $N:=\nu_{1}+\cdots+\nu_{n}$ zeros in $[a, b), M_{0}(t)$ must have at least $N$ zeros in $[a, b)$. Applying again Lemma 7 , we see that

$$
Z\left(M_{0} ;[a, b)\right) \leqslant n-1+\nu_{1}-1+\cdots+\nu_{n}-1=N-1
$$

or $M_{0}(\mathbf{x} ; t) \equiv 0$. This contradiction completes the proof of the lemma.

Remark 3. We showed in the proof of Lemma 1 that the equalities $a_{k, \nu_{k}-1}=0$ for $k=2, \ldots, n$ imply (6) in the case $\alpha=0$. By the same argument, one can verify that this holds for $0<\alpha \leqslant 1$ too. But, for $0<\alpha<1, F_{\alpha}(\mathbf{x} ; t)$ does not vanish identically on any subinterval, and $F_{\alpha}(\mathbf{x} ; t)$ has only $\nu_{1}+\cdots+\nu_{n}$ zeros: $\left\{x_{k}\right\}_{1}^{n}$ of multiplicities $\left\{v_{k}\right\}_{1}^{n}$, respectively. Therefore, $F_{\alpha}^{\left(\nu_{1}\right)}\left(x_{1}\right) \neq 0$. Hence, the relations $a_{k, v_{k}-1}=0$ for $k=2, \ldots, n$ imply $a_{1, \nu_{1}-1}=0$ for $0<\alpha<1$ and $1<p<\infty$. 
Proof of Lemma 2. Assuming the contrary, there exist sequences $\left\{\alpha_{m}\right\}_{0}^{\infty},\left\{\mathbf{x}_{m}\right\}_{1}^{\infty}$,

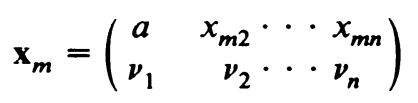

such that

$$
\Phi\left(\alpha_{m} ; \overline{\mathbf{x}}_{m}\right)=\overline{0}, \quad \bar{x}_{m}:=\left(x_{m 2}, \ldots, x_{m n}\right)
$$

for $m=1,2, \ldots, \alpha_{m} \rightarrow \alpha_{0} \in[0,1]$ and $x_{m}$ tends to some

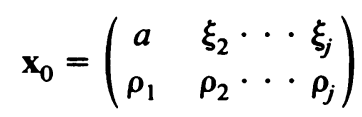

with $a<\xi_{2}<\cdots<\xi_{j}<b, j<n$, as $m \rightarrow \infty$. Remark that $\Phi\left(0 ; \bar{x}_{m}\right)=\overline{0}$ only for $\bar{x}_{m}=\bar{y}:=\left(y_{2}, \ldots, y_{n}\right)$, according to Lemma 1 . Therefore, we may assume without loss of generality that $\alpha_{m} \neq 0, m=1,2, \ldots$

We know from Lemma 5 that $M_{\alpha_{m}}\left(\mathbf{x}_{m} ; t\right)$ converges uniformly to $M_{\alpha_{0}}\left(\mathrm{x}_{0} ; t\right)$ on any closed subset of $[a, b)$ which does not contain a breakpoint of $M_{\alpha_{0}}\left(\mathbf{x}_{0} ; t\right)$. It is not difficult to deduce from Lemma $6 \mathrm{~b}$ that $M_{\alpha_{0}}\left(\mathrm{x}_{0} ; t\right)$ is a continuous function. Indeed, each monospline $M_{\alpha_{m}}\left(\mathbf{x}_{m} ; t\right)$ has a maximal number of zeros. Further, it follows from the assumption $\Phi\left(\alpha_{m} ; \bar{x}_{m}\right)=\overline{0}$ and Remark 3 that the knots $\left\{x_{m i}\right\}_{i=1}^{n}$ of $M_{\alpha_{m}}\left(x_{m} ; t\right)$ have multiplicities $\left\{\nu_{i}-1\right\}$, respectively, i.e., odd numbers. Then, by Lemma $6 \mathrm{~b}$, the coefficients of each polynomial fragment of $M_{\alpha_{m}}\left(\mathbf{x}_{m} ; t\right)$ are bounded by a constant which does not depend on $m$. Consequently, $\left\|M_{\alpha_{m}}^{\prime}\left(\mathbf{x}_{m} ; \cdot\right)\right\|_{C[a, b]}$ is bounded. This implies the continuity of $M_{\alpha_{0}}\left(\mathbf{x}_{0} ; t\right)$.

Next, we prove that $F_{\alpha_{0}}\left(\mathrm{x}_{0} ; t\right)$ does not vanish identically on any interval. First, note that $F_{\alpha_{0}}\left(\mathrm{x}_{0} ; t\right)$ is not zero identically, since

$$
F_{\alpha_{0}}^{(r)}\left(\mathbf{x}_{0} ; t\right)=\left|M_{\alpha_{0}}\left(\mathbf{x}_{0} ; t\right)\right|^{p-2} M_{\alpha_{0}}\left(\mathrm{x}_{0} ; t\right)
$$

and $M_{\alpha_{0}}\left(\mathrm{x}_{0} ; t\right) \equiv 0$ would imply $\alpha_{0}=0, y_{k} \in\left\{\xi_{2}, \ldots, \xi_{j}\right\}$ for $k=2, \ldots, n$ which is obviously impossible because $j<n$. Now assume that $F_{\alpha_{0}}\left(\mathbf{x}_{0} ; t\right)$ vanishes identically on some subinterval $\left[\tau_{1}, t_{1}\right]$ of $[a, b)$. Let $t_{2}$ be the next point after $t_{1}$ which is a zero of $F_{\alpha_{0}}\left(\mathrm{x}_{0} ; t\right)$ of multiplicity at least $r$ (note that $\tau_{1}$ may play the role of $t_{2}$ since we are thinking of $[a, b)$ as a circle). The function $F_{\alpha_{0}}\left(\mathrm{x}_{0} ; t\right)$ has at least $2 r+N_{1}$ zeros in $\left[t_{1}, t_{2}\right]$ where $N_{1}$ is the sum of those $\left\{\rho_{k}\right\}_{1}^{j}$ for which $\xi_{k} \in\left(t_{1}, t_{2}\right)$. According to the choice of $t_{2}, \rho_{k}<r$ for these $\xi_{k}$. By Rolle's theorem, $M_{\alpha_{0}}\left(x_{0} ; t\right)$ has at least $r+N_{1}$ sign changes in $\left(t_{1}, t_{2}\right)$. Moreover, $M_{\alpha_{0}}\left(\mathrm{x}_{0} ; t_{1}\right)=0$ since $M_{\alpha_{0}}\left(\mathbf{x}_{0} ; t\right) \equiv 0$ on $\left(\tau_{1}, t_{1}\right)$ and $M_{\alpha_{0}}\left(\mathbf{x}_{0} ; t\right)$ is continuous. Therefore $M_{\alpha_{0}}\left(\mathbf{x}_{0} ; t\right)$ has at least $r+1+N_{1}$ zeros in $\left[t_{1}, t_{2}\right)$. This contradicts Lemma $8 \mathrm{a}$ in the case $0<\alpha_{0}<$ 1. If $\alpha_{0}=0$ we apply again Rolle's theorem and conclude that $M_{0}^{\prime}\left(\mathrm{x}_{0} ; t\right)$ has $r+N_{1}$ sign changes in $\left(t_{1}, t_{2}\right)$. Then the function

$$
\alpha\left\{\frac{(b-t)^{r-1}}{(r-1) !}-\sum_{y_{k} \in\left(t_{1}, t_{2}\right)} \frac{\left(y_{k}-t\right)_{+}^{r-2}}{(r-2) !}\right\}+M_{0}^{\prime}\left(\mathrm{x}_{0} ; t\right)
$$

would have $r+N_{1}$ zeros in $\left(t_{1}, t_{2}\right)$ for small $\alpha$. We obtain a contradiction with Lemma 8a. Thus, $F_{\alpha_{0}}\left(\mathrm{x}_{0} ; t\right)$ does not vanish identically on any subinterval of $[a, b)$.

In the same way one can prove that $F_{\alpha_{0}}\left(\mathrm{x}_{0} ; t\right)$ does not have a zero of multiplicity $>r+1$, i.e., that $x_{0}$ does not have a coordinate $\xi_{i}$ of multiplicity $\rho_{i}>r+1$. Note that the assumption $\rho_{i} \geqslant r+1$ yields that $F_{\alpha_{0}}\left(\mathrm{x}_{0} ; t\right)$ has a zero at $\xi_{i}$ of multiplicity at least $r+1$ because $F_{\alpha_{0}}^{(r)}\left(\mathrm{x}_{0} ; t\right)$ is a continuous function.) Therefore, we can 
assume that $\rho_{i}<r$ for $i=1, \ldots, j$. Then $F_{\alpha_{0}}\left(\mathrm{x}_{0} ; t\right)$ has a zero at $\xi_{i}$ of multiplicity at least $\rho_{i}$. On the other hand, $M_{\alpha_{0}}\left(\mathrm{x}_{0} ; t\right)$ has a knot at $\xi_{i}$ of multiplicity at most $\rho_{i}-l_{i}$, where $l_{i}$ is the number of those coordinates of $\mathbf{x}_{m}$ which tend to $\xi_{i}$ as $m \rightarrow \infty$. Hence $F_{\alpha_{0}}\left(\mathrm{x}_{0} ; t\right)$, and, consequently, $M_{\alpha_{0}}\left(\mathrm{x}_{0} ; t\right)$ has at least $\rho_{1}+\cdots+\rho_{j}$ $=\nu_{1}+\cdots+\nu_{n}=N$ zeros in $[a, b)$. On the other hand,

Case I. For $0<\alpha_{0}<1$, by Lemma $8 \mathrm{~b}$,

$$
Z\left(M_{0}\left(\mathbf{x}_{0} ; \cdot\right) ;[a, b)\right)<\sum_{i=1}^{j}\left(\rho_{i}-l_{i}+1\right)=N-\left(l_{1}+\cdots+l_{j}\right)+j<N-1
$$

since $l_{1}+\cdots+l_{j}=n>j$. We obtain a contradiction;

Case II. For $\alpha_{0}=0$, by Lemma 7,

$$
\begin{aligned}
Z\left(M_{0}\left(\mathrm{x}_{0} ; \cdot\right) ;[a, b)\right) & <n-1+\sum_{i=1}^{j}\left(\rho_{i}-l_{i}\right) \\
& =n-1+N-\left(l_{1}+\cdots+l_{j}\right)=N-1
\end{aligned}
$$

a contradiction.

Therefore $j=n$. The lemma is proved.

Proof of Lemma 3. First we shall show that (3.1) holds for $p=2$. To this end, we construct the unique natural spline function $\varphi_{i}(\bar{x} ; t) \in \mathscr{T}_{2 r-1}(1 ; \mathbf{x})$ where $\bar{x}=$ $\left(x_{2}, \ldots, x_{n}\right) \in \Omega_{e}, \mathbf{x}=\left(\begin{array}{c}x_{1} \cdots x_{n} \\ v_{1} \cdots v_{n}\end{array}\right)$ which satisfies the interpolation conditions

$$
\left\{\begin{array}{l}
\varphi_{i}^{(j)}(\bar{x} ; a)=\varphi_{i}^{(j)}(\bar{x} ; b), \quad j=0, \ldots, r-1, \\
\varphi_{i}^{(\lambda)}\left(\bar{x} ; x_{k}\right)=0, \quad k \neq i, \lambda=0, \ldots, \nu_{k}-1, \\
\varphi_{i}^{(\lambda)}\left(\bar{x} ; x_{i}\right)=0, \quad \lambda=0, \ldots, \nu_{i}-2, \\
\varphi_{i}^{\left(\nu_{i}-1\right)}\left(\bar{x} ; x_{i}\right)=1, \\
\varphi_{i}^{(r+j)}(\bar{x} ; a+)=\varphi_{i}^{(r+j)}(\bar{x} ; b-), \quad j=0, \ldots, r-\nu_{1}-1 .
\end{array}\right.
$$

According to Corollary 1 , the rule based on the coefficients $\left\{a_{k \lambda}\right\}$ of the spline $M_{0}(\mathbf{x} ; t)$ is exact for $\varphi_{i}(\bar{x} ; t)$. Therefore

$$
b_{i}(0 ; \bar{x})=L_{0}\left(\varphi_{i}(\bar{x} ; \cdot)\right)=\sum_{k=2}^{n} \varphi_{i}\left(\bar{x} ; y_{k}\right) .
$$

We know from Proposition 1 that the determinant of the system (7) is nonsingular at every $\bar{x} \in \Omega_{e}$. Hence, by the implicit function theorem, the coefficients of the spline $\varphi_{i}(\bar{x} ; t)$ are continuous functions of $\bar{x}$ in $\Omega_{e}$. Then

$$
\left\|\varphi_{i}^{(\lambda)}(\bar{x} ; \cdot)-\varphi_{i}^{(\lambda)}(\bar{y} ; \cdot)\right\|_{C[a, b]} \rightarrow 0, \quad \lambda=0, \ldots, r-1,
$$

as $\bar{x} \rightarrow \bar{y}$. Now it is easy to see that

$$
b_{i}(0 ; \bar{x})=\frac{\left(y_{i}-x_{i}\right)^{\nu_{i}-1}}{\left(\nu_{i}-1\right) !}+\sum_{k=2}^{n} o\left(\left|y_{k}-x_{k}\right|^{p_{k}-1}\right)
$$

for each $\bar{x}=\left(x_{2}, \ldots, x_{n}\right)$ near to $\bar{y}$. Indeed, by Taylor's formula

$$
\varphi_{i}\left(\bar{x} ; y_{k}\right)=\left(y_{k}-x_{k}\right)^{\nu_{k}-1} \varphi_{i}^{\left(\nu_{k}-1\right)}\left(\bar{x} ; \eta_{k}\right) /\left(\nu_{k}-1\right) \text { ! }
$$

where $\eta_{k}$ lies between $x_{k}$ and $y_{k}$. Since, according to (9),

$$
\varphi_{i}^{\left(\nu_{k}-1\right)}\left(\bar{x} ; \eta_{k}\right) \rightarrow \varphi_{i}^{\left(\nu_{k}-1\right)}\left(\bar{y} ; y_{k}\right)= \begin{cases}0 & \text { if } i \neq k, \\ 1 & \text { if } i=k,\end{cases}
$$


as $\bar{x} \rightarrow \bar{y}$, the relation (10) is clear. (Remember that $\left\{\nu_{k}\right\}_{1}^{n}$ are even natural numbers, hence $\nu_{k}-1>1$.)

Now we make the invertible, orientation-preserving change of variables $z_{i}=$ $\left(x_{i}-y_{i}\right)^{v_{i}-1} /\left(\nu_{i}-1\right) !, i=2, \ldots, n$, and get

$$
b_{i}(0 ; \bar{x})=-z_{i}+\sum_{k=2}^{n} o\left(\left|z_{i}\right|\right)=: \tilde{b}_{i}(\bar{z})
$$

Let us set $B(\bar{z}):=\left(\tilde{b}_{2}(\bar{z}), \ldots, \tilde{b}_{n}(\bar{z})\right)$. Clearly

$$
\operatorname{det}\left(\frac{\partial B(\overline{0})}{\partial \bar{z}}\right)=(-1)^{n-1}
$$

By the implicit function theorem, $B(\bar{z})$ is $1-1$ for $\bar{z}$ near $\overline{0}$ and hence $\Phi(0 ; \bar{x})$ is $1-1$ for $\bar{x}$ near $\bar{y}$, say in the neighborhood $U(\bar{y}) \subset \Omega_{e}$ of $\bar{y}$. Let $\bar{\xi}=\left(\xi_{2}, \ldots, \xi_{n}\right) \in U(\bar{y})$ and denote $\bar{c}=\Phi(0 ; \bar{\xi})$. Then the equation $\Phi(0 ; \bar{x})=\bar{c}$ has a unique solution (which is $\bar{\xi}$ ) in $U(\bar{y})$. Therefore, by (i),

$$
\operatorname{deg}(\Phi(0 ; \cdot), U(\bar{y}), \bar{c})=\operatorname{sign} \operatorname{det}\left(\frac{\partial \Phi(0 ; \bar{\xi})}{\partial \bar{x}}\right)
$$

But the latter determinant has a dominant main diagonal for $\bar{\xi}$ near $\bar{y}$ and evidently

$$
\text { sign } \operatorname{det}\left(\frac{\partial \Phi(\overline{0} ; \bar{\xi})}{\partial \bar{x}}\right)=(-1)^{n-1} \operatorname{sign} \prod_{k=2}^{n}\left(y_{k}-\xi_{k}\right)^{\nu_{k}-2}=(-1)^{n-1}
$$

since $\left\{\nu_{k}\right\}_{2}^{n}$ are even. On the other hand, $\bar{c}(\bar{\xi})$ tends to $\overline{0}$ as $\bar{\xi} \rightarrow \bar{y}$ because $\Phi(0 ; \cdot)$ is a continuous function and $\Phi(0 ; \bar{y})=\overline{0}$. It remains to apply (iv) with respect to a sequence of points $\bar{c}(\bar{\xi})$ with $\bar{\xi} \rightarrow \bar{y}$ to obtain that

$$
\operatorname{deg}(\Phi(0 ; \cdot), U(\bar{y}), \overline{0})=(-1)^{n-1} .
$$

But, according to Lemma $1, \bar{y}$ is the unique solution of the equation $\Phi(0 ; \bar{x})=\overline{0}$ in $\Omega_{\varepsilon^{*}}$. Therefore

$$
\operatorname{deg}\left(\Phi(0 ; \cdot), \Omega_{e}, \overline{0}\right)=\operatorname{deg}(\Phi(0 ; \cdot), U(\bar{y}), \overline{0})=(-1)^{n-1} .
$$

The relation (3.1) is proved for $p=2$.

Now suppose that $p^{*}$ is an arbitrary number fixed in $(1, \infty)$. Consider the mapping $\Phi_{\beta}:[0,1] \times \Omega_{\varepsilon} \rightarrow \mathbf{R}^{n-1}$ defined by the equality $\Phi_{\beta}(\bar{x})=$ $\left(b_{2}(0 ; \bar{x}), \ldots, b_{n}(0 ; \bar{x})\right)$ where the parameter $p$ in the definition of the coefficients $b_{k}(0 ; \bar{x})$ is chosen to be $\beta p^{*}+2(1-\beta), \beta \in[0,1]$. We have proved in Lemma 1 that $\bar{x}=\bar{y}$ is the only solution of the equation $\Phi(0 ; \bar{x})=\overline{0}$ in $\Omega_{e}$ for each $p \in(1, \infty)$, i.e., the equation $\Phi_{\beta}(\bar{x})=\overline{0}$ has a unique solution $\bar{x}=\bar{y}$ in $\Omega_{e}$ for $\beta \in[0,1]$. Since $\bar{y} \notin \partial \Omega_{e}$, the property (iii) yields

$$
\operatorname{deg}\left(\Phi_{1}, \Omega_{e}, \overline{0}\right)=\operatorname{deg}\left(\Phi_{0}, \Omega_{e}, \overline{0}\right)=(-1)^{n-1} .
$$

The lemma is proved.

It remains to prove Lemma 4. Its proof is based on the following interpolation theorem for natural splines with weight. 
THEOREM 6. Let

$$
\xi=\left(\begin{array}{c}
\xi_{1} \cdots \xi_{m} \\
\mu_{1} \cdots \mu_{m}
\end{array}\right), \quad \mathbf{x}=\left(\begin{array}{c}
x_{1} \cdots x_{n} \\
\nu_{1} \cdots \nu_{n}
\end{array}\right)
$$

be arbitrary systems of points satisfying the conditions

$$
\begin{aligned}
& a \leqslant \xi_{1}<\cdots<\xi_{m}<b, \quad a<x_{1}<\cdots<x_{n}<b ; \\
& 0 \leqslant \nu_{k}<r, \quad k=1, \ldots, n, \\
& 0 \leqslant \mu_{i}<r, \quad i=1, \ldots, m .
\end{aligned}
$$

The number $\nu_{1}+\cdots+\nu_{n}$ is odd; $\nu_{1}+\cdots+\nu_{n}=\mu_{1}+\cdots+\mu_{m}$. Let us think of the points $\left\{\xi_{i}\right\}_{1}^{m}$ and $\left\{x_{k}\right\}_{1}^{n}$ as being given on a circle obtained by joining the endpoints $a$ and $b$ of the interval $[a, b]$. Suppose that $\mathbf{x}$ and $\xi$ satisfy the requirement:

For any pair $\left(\xi^{\prime}, \xi^{\prime \prime}\right)$ from the set $\left\{\xi_{1}, \ldots, \xi_{m}\right\}, 1+\nu_{i_{1}}+\cdots+\nu_{i_{k}}>\mu_{j_{1}}$ $+\cdots+\mu_{j i}$, provided the points $x_{i_{1}}, \ldots, x_{i_{k}}$ and $\xi_{j_{1}}, \ldots, \xi_{j_{i}}$ lie between $\xi^{\prime}$ and $\xi^{\prime \prime}$ (i.e., on the left-right oriented arc $\left.\left(\xi^{\prime}, \xi^{\prime \prime}\right)\right)$.

Suppose that $\omega$ is an arbitrary $(b-a)$-periodic weight in $[a, b]$. Then, for every function $f \in \tilde{C}^{r-1}[a, b]$ there exists a unique natural spline $S \in \Re_{2 r-1}(\omega ; \xi)$ satisfying the interpolation conditions

$$
S^{(\lambda)}\left(x_{k}\right)=f^{(\lambda)}\left(x_{k}\right), \quad k=1, \ldots, n, \lambda=0, \ldots, \nu_{k}-1 .
$$

Proof. Assuming the contrary, there exists a function $S \neq 0, S \in \Re_{2 r-1}(\omega ; \xi)$ satisfying the homogeneous system

$$
\left\{\begin{array}{l}
S^{(j)}(a)-S^{(j)}(b)=0, \quad j=0, \ldots, r-1, \\
S^{(\lambda)}\left(x_{k}\right)=0, \quad k=1, \ldots, n, \lambda=0, \ldots, \nu_{k}-1, \\
g^{(j)}(a+)-g^{(j)}(b-)=0, \quad j=0, \ldots, r-\mu-1,
\end{array}\right.
$$

where

$$
\mu= \begin{cases}0 & \text { if } a \neq \xi_{1}, \\ \mu_{1} & \text { if } a=\xi_{1} .\end{cases}
$$

Suppose that $S$ vanishes identically on some subinterval. Then there exists an arc $\left[t_{1}, t_{2}\right]$ such that $S(t)=0$ if $t \in\left[t_{1}-\varepsilon, t_{1}\right] \cup\left[t_{2}, t_{2}+\varepsilon\right]$ for some $\varepsilon>0$ and $S(t) \neq$ 0 almost everywhere in $\left[t_{1}, t_{2}\right]$. Clearly $\left(t_{1}, t_{2}\right)=\left(\xi^{\prime}, \xi^{\prime \prime}\right)$ for some $\xi^{\prime}, \xi^{\prime \prime} \in$ $\left\{\xi_{1}, \ldots, \xi_{m}\right\}$. Let $x_{i_{1}}, \ldots, x_{i_{k}}$ be the points from $\mathbf{x}$ which lie between $\xi^{\prime}$ and $\xi^{\prime \prime}$. Then $S(t)$ has at least $2 r+\nu_{i_{1}}+\cdots+v_{i_{k}}$ zeros in $\left[t_{1}, t_{2}\right]: t_{1}, x_{i_{1}}, \ldots, x_{i_{k}}, t_{2}$ of multiplicities $r, \nu_{i_{1}}, \ldots, \nu_{i_{k}}, r$, respectively. By Rolle's theorem, $S^{(r)}(t)$ and, consequently, $g(t)$ must have $r+\nu_{i_{1}}+\cdots+\nu_{i_{k}}$ zeros at least in $\left(t_{1}, t_{2}\right)$. But $g(t)=0$ on $\left[t_{1}-\varepsilon, t_{1}\right] \cup\left[t_{2}, t_{2}+\varepsilon\right]$ and $g(t)$ is continuous at $t_{1}$, because $t_{1}=\xi^{\prime}$ and $\mu_{j}<r$, $j=1, \ldots, m$. Therefore $g\left(t_{1}\right)=0$. Thus $g(t)$ has $r+1+\nu_{i_{1}}+\cdots+\nu_{i_{k}}>r+\mu_{j_{1}}$ $+\cdots+\mu_{j,}$ zeros in $\left[t_{1}, t_{2}\right]$, where $\xi_{j,}, \ldots, \xi_{j,}$ are the points of $\xi$ which lie in $\left(\xi^{\prime}, \xi^{\prime \prime}\right) .$, On the other hand, according to a known result by Schumaker [16], $Z\left(g ;\left[t_{1}, t_{2}\right]\right)<r-1+\mu_{j_{1}}+\cdots+\mu_{j_{i}}$. This contradiction shows that $S$ has only isolated zeros in $[a, b)$.

Since $S$ satisfies (11), by Rolle's theorem, $g(t)$ must have $N:=\nu_{1}+\cdots+\nu_{n}$ zeros at least in $[a, b)$. But $\nu_{1}+\cdots+\nu_{n}=\mu_{1}+\cdots+\mu_{m}$ and it is an odd number. Then, by Lemma $7, Z(g ;[a, b)) \leqslant N-1$, a contradiction. The theorem is proved. 
Proof of Lemma 4. It is easily seen that for given $j$ and $k$ the multiplicities $\left\{\hat{\nu}_{i}\right\}_{1}^{n}$, $\left\{\hat{\mu}_{i}\right\}_{1}^{n}$, where

$$
\hat{\nu}_{i}:=\left\{\begin{array}{ll}
\nu_{i} & \text { if } i \neq j, \\
\nu_{i}-1 & \text { if } i=j,
\end{array} \quad \hat{\mu}_{i}:= \begin{cases}\nu_{i} & \text { if } i \neq k, \\
\nu_{i}-1 & \text { if } i=k,\end{cases}\right.
$$

and the points $\mathrm{x}=\left(\left(x_{1}, \hat{\nu}_{1}\right), \ldots,\left(x_{n}, \hat{\nu}_{n}\right)\right), \xi=\left(\left(x_{1}, \hat{\mu}_{1}\right), \ldots,\left(x_{n}, \hat{\mu}_{n}\right)\right)$ satisfy the requirements of Theorem 6 . Therefore,

$$
\operatorname{det} \Delta_{j k}(\omega ; \mathbf{x}) \neq 0
$$

Suppose that the nodes $\mathbf{x}$ are fixed and $\omega_{1}, \omega_{2}$ are two $(b-a)$-periodic weights. Then the function $\omega(\delta ; t):=\delta \omega_{1}(t)+(1-\delta) \omega_{2}(t)$ is a weight too, for each $\delta \in[0,1]$. Therefore, in view of $(12), \operatorname{det} \Delta_{j k}(\omega(\delta ; \cdot), \mathbf{x}) \neq 0$ for every $\delta \in[0,1]$. Since the determinant is a continuous function of $\delta$, we conclude that sign $\operatorname{det} \Delta_{j k}\left(\omega_{1} ; \mathbf{x}\right)=\operatorname{sign} \operatorname{det} \Delta_{j k}\left(\omega_{2} ; \mathbf{x}\right)$. Thus, it suffices to prove the lemma only for $\omega(t) \equiv 1$.

Let $D\left(\left.f\right|_{\alpha_{1} \cdots \alpha_{l}} ^{t_{1} \cdots t_{l}}\right)$ denote the divided difference of $f$ at the points $t_{1}<\cdots<t_{l}$ of multiplicities $\alpha_{1}, \ldots, \alpha_{l}$, respectively. It is well known that

$$
D\left(\left.f\right|_{a_{1} \cdots \alpha_{l}} ^{t_{1} \cdots t_{l}}\right)=f^{(N)}(\eta) / N !, \quad \eta \in\left(t_{1}, t_{l}\right),
$$

provided $f \in C^{N}[a, b], \alpha_{1}+\cdots+\alpha_{l}=N+1, t_{i} \in[a, b], i=1, \ldots, l$. It can be verified (see Čakalov [7, Theorem 1]) that

$$
D\left(\left.f\right|_{m, 1} ^{\tau, \tau+h}\right)=\frac{1}{h^{m}} f(\tau+h)-\sum_{\lambda=0}^{m-1} \frac{1}{\lambda ! h^{m-\lambda}} f^{(\lambda)}(\tau) .
$$

We shall show that

$$
\operatorname{sign} \Delta_{j k}(1 ; \mathbf{x})=\operatorname{sign} \Delta_{j+1, k}(1 ; \mathbf{x}), \quad j=1, \ldots, n-1,
$$

for every fixed $k \in\{2, \ldots, n\}$. Then (15) will imply the assertion of the lemma.

Let $k$ be fixed. Let $M(k ; t)$ stand for a monospline of degree $r$ with knots $\left(x_{1}, \nu_{1}\right), \ldots,\left(x_{k}, \nu_{k}-1\right), \ldots,\left(x_{n}, \nu_{n}\right)$. Denote by $\Delta(\cdot)$ the matrix corresponding to the system of the form (2.8) with interpolating nodes those indicated in the parentheses and with a $F$ defined by $M(k ; t)$. For instance, $\Delta_{j k}(1 ; \mathbf{x})$ can be rewritten as

$$
\Delta\left(\begin{array}{c}
x_{1} \cdots x_{j} \cdots x_{n} \\
\nu_{1} \cdots \nu_{j}-1 \cdots v_{n}
\end{array}\right)
$$

Suppose that $0<h<x_{j+1}-x_{j}$. Consider the determinant of the matrix

$$
\Delta_{j k}^{h}:=\Delta\left(\begin{array}{ccc}
x_{1} \cdots x_{j} & x_{j}+h & x_{j+1} \cdots x_{n} \\
\nu_{1} \cdots \nu_{j}-1 & 1 & \nu_{j+1}-1 \cdots \nu_{n}
\end{array}\right) .
$$

It follows from Theorem 6 that det $\Delta_{j k}^{h} \neq 0$ for each $h, 0<h<x_{j+1}-x_{j}$. We shall prove that sign det $\Delta_{j k}^{h}=\operatorname{sign} \operatorname{det} \Delta_{j+1, k}$. In order to do this, let us change $\Delta_{j k}^{h}$ in the following way:

Multiply the $r+\nu_{1}+\cdots+\nu_{j}$ th row by $\left(\nu_{j}-1\right) ! / h^{\nu_{j}-1}$ and add to it the sum of the rows with numbers $r+\nu_{1}+\cdots+\nu_{j-1}+\lambda+1, \lambda=0, \ldots, \nu_{j}-2$, multiplied by $-\left(\nu_{j}-1\right) ! /\left(\lambda ! h^{\nu_{j}-1-\lambda}\right)$, respectively. The new matrix $\tilde{\Delta}_{j k}^{h}$ will correspond 
to the system which is obtained from the previous one, changing the $r+\nu_{1}$ $+\cdots+\nu_{j}$ th equation by

$$
\left(\nu_{j}-1\right) ! D\left(\left.F\right|_{v_{j},-1,1} ^{x_{j}, x_{j}+h}\right)=0 .
$$

Since $\nu_{j}<r,(13)$ implies

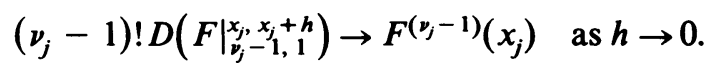

Therefore,

$$
\operatorname{det} \tilde{\Delta}_{j k}^{h} \rightarrow \operatorname{det} \Delta_{j+1, k} \quad \text { as } h \rightarrow 0 \text {. }
$$

But det $\tilde{\Delta}_{j k}^{h}=\left(\left(\nu_{j}-1\right) ! / h^{\nu_{j}-1}\right) \operatorname{det} \Delta_{j k}^{h}$ and $\operatorname{det} \Delta_{j+1, k} \neq 0$. Hence sign $\operatorname{det} \Delta_{j k}^{h}=$ sign det $\Delta_{j+1, k}$ for small $h$. We have already mentioned that $\operatorname{det} \Delta_{j k}^{h} \neq 0$ for $0<h<x_{j+1}-x_{j}$. Therefore, sign det $\Delta_{j k}^{h}=\operatorname{sign} \operatorname{det} \Delta_{j+1, k}$ for all $h, 0<h<x_{j+1}$ $-x_{j}$.

In the same way, using the explicit expression of $D\left(\left.f\right|_{1, m} ^{\tau-h, \tau}\right)$ one can show that sign det $\Delta_{j k}^{h}=$ sign det $\Delta_{j k}$. The relation (15) is proved. This completes the proof of the lemma.

Acknowledgement. The author is grateful to the referees for their helpful remarks, particularly for those concerning the organization of the paper.

Department of Mathematics

University of Sofia

Boulevard Anton Ivanov 5

1126 Sofia, Bulgaria

1. N. I. AHIEZER, Lectures on Approximation Theory, "Nauka", Moscow, 1965.

2. R. B. BArRar \& H. L. LOEB, "On monosplines with odd multiplicities of least norm," J. Analyse Math., v. 33, 1978, pp. 12-38.

3. D. BARRow, "On multiple node Gaussian quadrature formulae," Math. Comp., v. 32, 1978, pp. 431-439.

4. R. Bellman, "On the positivity of determinants with dominant main diagonal," J. Math. Anal. Appl., v. 59, 1977, p. 210.

5. B. D. Bojanov, "Existence and characterization of monosplines of least $L_{p}$ deviation," Constructive Function Theory '77, Sofia, 1980, pp. 249-268. BAN.

6. B. D. Bojanov, "Uniqueness of the monosplines of least deviation," Numerische Integration, ISNM 45, Birkhäuser-Verlag, Basel, 1979, pp. 67-97.

7. L. Cakalov, "On a representation of Newton's quotients in the interpolation theory and its applications," Anmuaire Univ. Sofia Fac. Math. Méc., v. 34, 1938, pp. 353-405.

8. K. JetTER \& G. LANGE, "Die Eindeutigkeit $L_{2}$-optimaler polynomialer Monosplines," Math. $Z$., v. 158, 1978, pp. 23-34.

9. R. S. Johnson, "On monosplines of least deviation," Trans. Amer. Math. Soc., v. 96, 1960, pp. 458-477.

10. A. A. Ligun, "Exact inequalities for spline functions and best quadrature formulae for certain classes of functions," Mat. Zametki, v. 19, 1979, pp. 913-926.

11. N. E LuŠPAI, "Best quadrature formulae for classes of differentiable periodic functions," Mat. Zametki, v. 6, 1969, pp. 475-482.

12. N. E. LušpA, "Optimal quadrature formulae for classes of functions with an $L_{p}$-integrable $r$-th derivative," Anal. Math., v. 5, 1979, pp. 67-88.

13. C. MICCHELLI, "The fundamental theorem of algebra for monosplines with multiplicities," Linear Operators and Approximation, ISNM v. 20, Birkhäuser-Verlag, Basel, 1972, pp. 419-430.

14. V. P. MotorNĭ, "On the best quadrature formula of the form $\sum_{k-1}^{n} P_{k} f\left(x_{k}\right)$ for certain classes of periodic differentiable functions," Izv. Akad. Nauk SSSR Ser. Mat., v. 38, 1974, pp. 583-614.

15. J. M. OrTegA \& W. C. RheInBoldt, Iterative Solution of Nonlinear Equations in Several Variables, Academic Press, New York, 1970. 
16. L. L. SCHUMaker, "Zeros of spline functions and applications," J. Approximation Theory, v. 18, 1976, pp. $152-168$.

17. J. T. SCHWARTZ, Nonlinear Functional Analysis, Gordon and Breach, New York, 1969.

18. A. A. ŻensYkBaev, "On the best quadrature formula on the class $W^{\prime} L_{p}$ ", Dokl. Akad. Nauk SSSR, v. 227,1976 , pp. $277-279$.

19. A. A. Zensykbaev, "Best quadrature formula for the class $W^{\prime} L_{2}$ " Anal. Math., v. 3, 1977, pp. 83-93.

20. A. A. ŻensYkBaev, "Best quadrature formula for certain classes of periodic functions," Izv. Akad. Nauk SSSR Ser. Mat., v. 41, 1977, pp. 1110-1124.

21. A. A. ŻenSYKBaEv, "Characteristic properties of the best quadrature formulae," Sibirsk. Mat. $Z$., v. 20, 1979, pp. $49-68$. 